\title{
A multistep docking and scoring protocol for congeneric series: Implementation on kinase DFG-out type II inhibitors
}

José M. Granadino-Roldán ${ }^{1,{ }^{*}}$, Andrés Garzón ${ }^{2}$, Patricia Gomez-Gutierrez ${ }^{3}$, Ignacio Pasamontes-Funez ${ }^{4}, \mathrm{M}$. Santos Tomas ${ }^{5}$, Jaime Rubio-Martinez ${ }^{4, *}$

${ }^{1}$ Departamento de Química Física, Facultad de Ciencias Experimentales, Universidad de Jaén, Campus "Las Lagunillas" s/n, 23071, Jaén, Spain

${ }^{2}$ Departamento de Química Física, Facultad de Farmacia, Universidad de Castilla-La Mancha, Paseo de los Estudiantes, s/n, 02071, Albacete, Spain

${ }^{3}$ Department of Chemical Engineering, Universitat Politecnica de Catalunya, ETSEIB. Av. Diagonal, 647, 08028 Barcelona, Spain

${ }^{4}$ Departament de Química Física, Universitat de Barcelona (UB) and the Institut de Recerca en Quimica Teorica i Computacional (IQTCUB), Martí i Franqués 1, 08028 Barcelona, Spain

${ }^{5}$ Department of Architecture Technology, Universitat Politecnica de Catalunya, Av. Diagonal 649, 08028 Barcelona, Spain

\section{Abstract}

Aim: Re-scoring of docking binding poses can significantly improve molecular docking results. Our aim was to evaluate post-processing docking protocols in order to determine the most suitable methodology for the study of the binding of congeneric compounds to protein kinases. Materials \& Methods: Diverse ligand-receptor poses generated after docking were submitted to different relaxation protocols. MMPB(GB)SA approach was applied for the evaluation of the binding affinity of complexes obtained. The performance of various MMPB(GB)SA methodologies was compared. Results: The inclusion of a post-processing protocol after docking enhances the quality of the results, although the best methodology is system-dependent. Conclusion: An examination of the interactions established has allowed us to suggest useful modifications for the design of new type II inhibitors.

\section{Keywords}

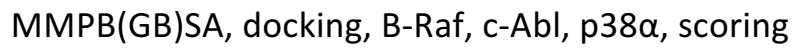

\section{Acknowledgments}

This work was supported by Ministerio Español de Ciencia y Tecnología (project CTQ2011-29285-C02-01 and 02), the Generalitat of Catalunya (project 2014SGR1017) and the Universidad de Jaén (Ayuda a Personal Investigador de la UJA para Estancias en Otros Centros de Investigación).

\footnotetext{
*E-mail:jmroldan@ujaen.es, jaime.rubio@ub.edu
} 


\section{Abstract}

Aim: Re-scoring of docking binding poses can significantly improve molecular docking results. Our aim was to evaluate post-processing docking protocols in order to determine the most suitable methodology for the study of the binding of congeneric compounds to protein kinases. Materials \& Methods: Diverse ligand-receptor poses generated after docking were submitted to different relaxation protocols. MMPB(GB)SA approach was applied for the evaluation of the binding affinity of complexes obtained. The performance of various MMPB(GB)SA methodologies was compared. Results: The inclusion of a post-processing protocol after docking enhances the quality of the results, although the best methodology is system-dependent. Conclusion: An examination of the interactions established has allowed us to suggest useful modifications for the design of new type II inhibitors. 
More than 500 protein kinases compose the so-called human kinome, whose members play a fundamental role in signalling pathways needed to control cellular processes such as metabolism, transcription, cell cycle progression, cytoskeletal rearrangement, apoptosis and differentiation [1]. Kinases transfer the $\psi$-phosphate of ATP to a substrate protein, regulating this way the activity of many enzymes. Dysfunction of kinases has been associated with a number of diseases, among them remarkably cancer [2]. The inhibition of deregulated protein kinases has thus been proposed as a target against cancer, and considerable efforts are being dedicated to develop selective small molecule kinase inhibitors [3]. A way to impede $\gamma$-phosphate transfer is to prevent ATP from binding to the kinase in its conserved ATP binding domain.

Kinase inhibitors can be classified depending on where they bind and how they block kinase catalysis. Most kinase inhibitors are ATP competitive, binding within the ATP binding site in the protein DFG-in active conformation, a cleft between the two lobes of the kinase catalytic domain. These are type I inhibitors, which typically lack selectivity against one particular kinase due to the highly conserved ATP binding site among the kinome [4]. To overcome this limitation, current drug discovery efforts aim to develop allosteric kinase inhibitors, most of them targeting the DFG-out inactive conformation. These DFG-out inhibitors stabilize the inactive conformation preventing ATP from binding [5]. When the inhibitor binds partially to the ATP binding site and partially to an allosteric site it is called a type II inhibitor, while compounds binding completely in allosteric sites are called type III inhibitors [6]. A unified view of allostery has been described recently [7].

Some allosteric inhibitors have been approved and others are in the early stages of clinical trials. Gleevec ${ }^{\circledR}$ was approved in 2002 for the treatment of chronic myeloid leukaemia and gastrointestinal stromal tumours, being the first compound proven to inhibit a kinase by binding the DFG-out allosteric site [8]. Nexavar ${ }^{\circledR}$ was approved in 2005 for the treatment of renal cell carcinoma and hepatocellular carcinomas, while BIRB-796, developed as a treatment for rheumatoid arthritis and Crohn's disease, was withdrawn from phase III clinical trials [9].

Due to the clinical importance of the abovementioned compounds there exists an enormous interest on the development of new similar derivatives able to overcome possible resistance and exhibiting higher selectivity. Thus, J. Dietrich et al. [10] synthesized 8 different combinations of the basic skeleton of Gleevec ${ }^{\circledR}$, Nexavar ${ }^{\circledast}$ and BIRB-796 and reported their inhibitory activity against the B-Raf, c-Abl and p38 $\alpha$ proteins. The analysis of the changes in inhibitory activity allowed the authors to extract some structure activity relationships, although unfortunately no X-ray structure of any of the proteins with the newly synthesized compounds has been reported up to date. This study aims to gain insight on the interactions established between these 8 new compounds and the 3 kinase proteins. The analysis of these interactions will lead to a deeper knowledge of the structure activity relationships and to propose further modifications to improve the inhibitors.

The usual approximation to predict the interaction mode between a ligand and a receptor makes use of docking methods based on the receptor structure, although it is well known that these methods suffer from serious deficiencies when trying to obtain a correlation between experimental affinity and docking score for a set of ligands $[11,12]$. One way to circumvent this problem arises when the studied compounds belong to a congeneric series, that is, they are formed from modifications to the same core, which allows to suppose that the core binding mode has to be the same and focus the effort during the docking procedure in generating and checking conformations for the noncommon part [13]. But even in these particular cases, there exist evidences showing that performing a re-scoring of the ligand-protein complexes obtained through docking, by means of a more accurate methodology, substantially improves the correlation between experimental and calculated results $[14,15]$. One of the most widely used approaches that combines computational efficiency and precision is the Molecular Mechanics Poisson Boltzmann (Generalized Born) Surface Area (MMPB(GB)SA) method [16]. Although this methodology is usually applied to a set of structures obtained from Molecular Dynamics (MD), it has been shown that its application to one structure is able to substantially improve docking results $[17,18]$. 
The dielectric constant, used by both GB and PB methods to determine the electrostatic contribution to binding, is divided into two parts, i.e. the external dielectric constant $\left(\varepsilon_{\text {ext }}\right)$ for the bulk solvent, which is a well-defined property, and the internal dielectric constant $\left(\varepsilon_{\text {int }}\right)$. The latter is not well defined because the ligand and protein are not uniform electrostatic media [19]. Moreover, it can be considered as a compensation factor for the interaction that is neglected in the continuum method [19], or just a parameter that depends on the method and system being used [20]. Although the original formulation of the MMPB(GB)SA methodology uses a value of 1 for $\varepsilon_{\text {int }}$ [16], it has been suggested that results could improve using a larger $\varepsilon_{\text {int }}$ [21], with optimum values of the interior dielectric constant varying from 1 to 25 [19]. The use of values lower than 1 can in fact be considered unrealistic taking into account its original physical definition, although if one focusses in considering it merely as a parameter to be adjusted some interesting conclusions could be extracted. Thus, in a recent article by Li et al. [22], the authors essay the use of a smooth dielectric function and vary what they define as the reference dielectric value from 0.1 to 4.0 .

Besides, it is well known that entropy calculations dominate the computational cost [23], so that entropy is sometimes calculated only for a subset of the snapshots from the molecular dynamics run. Although it is clear that the term is needed to obtain absolute binding free energies, it has also been reported not to improve the prediction when ranking within a given set $[24,25]$.

Hence, the aims of the present work are twofold. On the one hand, we present a multi-step protocol composed of an initial truncated docking and subsequent re-scoring with the MMPB(GB)SA methodology, being our goal to compare between a number of options in this protocol and decide the most appropriate methodology for the study of congeneric compounds of Nexavar ${ }^{\circledast}$, Gleevec ${ }^{\circledR}$ and BIRB-796. By means of different scoring functions we want to evaluate the performance of just using docking or re-scoring with different choices. Thus, we will compare re-scoring with MMGBSA (with different GB approaches), with MMPBSA (with different values for $\varepsilon_{\text {int }}$ ), and we will also check the influence of using one snapshot for MMPB(GB)SA or a short MD run. Finally, the influence of using an entropic term, given its computational cost, will also be evaluated.

On the other hand, apart from the analysis of methodologies, we plan, once the best method for each set is determined, analyse the proposed docked structures in order to gain insight into those interactions that could be improved and those that should be avoided to obtain improved allosteric inhibitors of these protein kinases.

\section{Materials \& Methods}

Minimizations, MD and MMPB(GB)SA calculations were carried out using the Amber ffg9SB force field for the proteins [26] and generalized amber force field (gaff) [27] for the ligands, as implemented in the Amber v.14 suite of programs [28]. Minimizations and MD runs were performed under periodic boundary conditions using the particle-mesh-Ewald method [29] for the treatment of the long-range electrostatic interactions. A cut-off distance of $10 \AA$ was chosen to compute the non-bonded interactions and the solvent was considered explicitly using TIP3P [30] water molecules with a minimum distance from the edge of the box of $15 \AA$ and removing those water molecules closer than $1.8 \AA$ from any protein atom. Counterions were added, when necessary, to neutralize the systems. A two steps heating protocol with an integration time step of $1 \mathrm{fs}$ has been used which includes increasing the temperature of the system to $300 \mathrm{~K}$ at a constant rate of $3 \mathrm{~K} \mathrm{ps}^{-1}$ during $100 \mathrm{ps}$ in the NVT ensemble, and $200 \mathrm{ps}$ at $300 \mathrm{~K}$ in the NPT ensemble to adjust density. After equilibration, a $2 \mathrm{fs}$ integration time step was used to run $2 \mathrm{~ns}$ of $\mathrm{MD}$ at $300 \mathrm{~K}$ in the NVT ensemble.

Preparation of the systems to work with Amber v.14 and post-processing of the minimization and MD runs used the Antechamber, LEaP, cpptraj and MMPBSA.py modules of AmberTools14 [28] package. Figures were rendered with UCSF Chimera [31], while graphs were prepared with Origin [32]. 
The five initial experimental X-Ray structures of the complexes c-Abl with Gleevec ${ }^{\circledR}$, B-Raf with Nexavar ${ }^{\circledR}$, p38 $\alpha$ with Gleevec $^{\circledR}$, p38 $\alpha$ with Nexavar $^{\oplus}$, and p38 $\alpha$ with BIRB-796 were obtained from the Protein Data Bank [33] (http://www.rcsb.org), with codes 1IEP [34], 1UWJ [35], 3HEC [36], 3HEG [36] and 1KV2 [37], respectively.

Missing residues for the three p38 $\alpha$ structures were taken from the solved structure with PDB code 1 W82 [38]. On the other hand, the B-Raf structure was modelled as described in reference [25]. Parameters and charges for all the ligands were obtained using Antechamber [28] with the gaff force field [27] and partial charges derived through the AM1-BCC method [39, 40], which is recommended in large-scale calculations because of its efficiency. All complexes were energy minimized to avoid possible steric clashes following a three steps protocol which first minimizes only water molecules and counterions (1000 steepest descent steps), later also side chains are allowed to move (1000 steepest descent steps), and ends with 5000 (steepest descent) steps allowing the whole system to move.

\section{Docking}

The docking procedure used as target proteins the five models prepared as explained before, and as ligands Gleevec ${ }^{\circledR}$, Nexavar ${ }^{\circledR}$ and BIRB-796, along with the 8 ligands synthesized by J. Dietrich et al. [10] (see Figure 1). Due to the fact that the ligands studied share a similar core (congeneric series, see Figure 2), we used a simplified approach for the conformational positional search of the ligands in the protein binding site. We assume that the common core of the ligands will interact with each protein in a similar way as the experimental one for each of the five receptors [13], without change to a different position. Thus, the substructures of the compounds highlighted in Figure 2 were superimposed, using a script written by the development team of the MOE software [41], to the experimental structures avoiding this way the placement stage during the docking process. After this, MOE [41] was used to generate the conformations of the rotatable bonds using the systematic approach and forbidding rotation for the common core substructure. Once the conformational ensemble for each ligand-protein complex was generated, the prediction of the ligand-protein binding energy was conducted with MOE [41], avoiding the placement stage and using as initial scoring function the Affinity dG energy function. Next, in order to introduce side chain flexibility in the receptor description, a Force Field post-placement methodology was used, which allows side chains located less than $10 \AA$ from the ligand to move freely. As a result, we obtained 55 (five X-Ray structures and eleven ligands) sets of ligand-protein conformations ranked in energy, each one composed of 20 different conformations. 


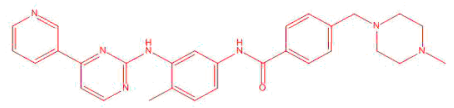

Gleevec $^{(\mathbb{R}} 1$

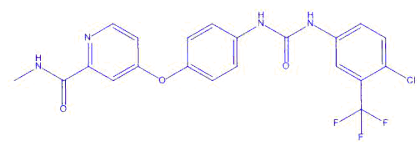

$\operatorname{Nexavar}^{\left({ }^{\circledR}\right)} \mathbf{2}$

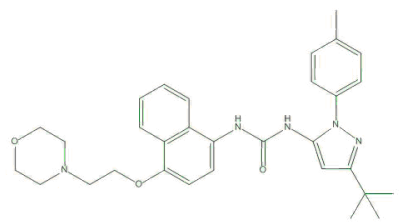

BIRB-796 3

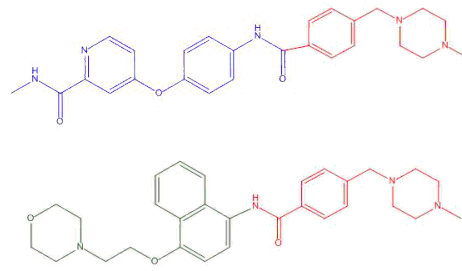

GleeAvar 4

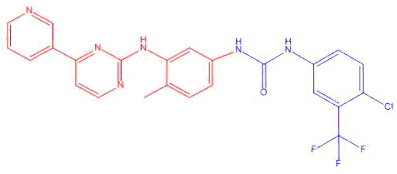

NexaVec 6

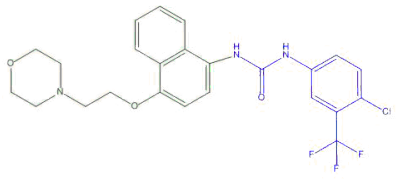

Nexa-796 7

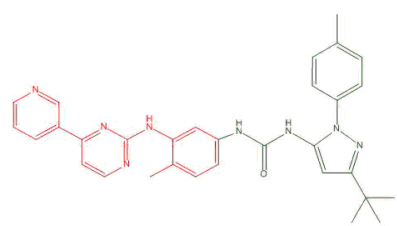

Gleev-796 5

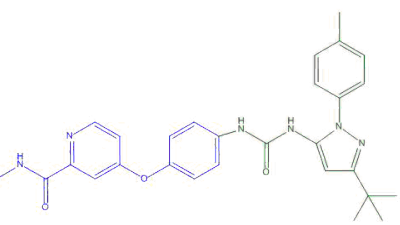

BIRvec 8
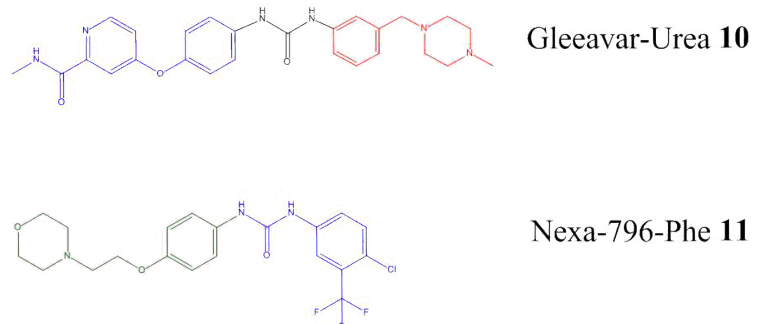

Nexa-796-Phe 11

Figure 1: Ligands studied in this work, highlighting with colours the origin of each part for those synthesized in reference [10] 


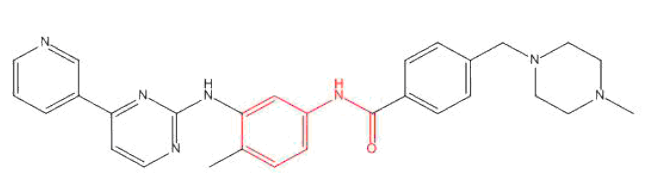

\section{Gleevec $^{\circledR} 1$}

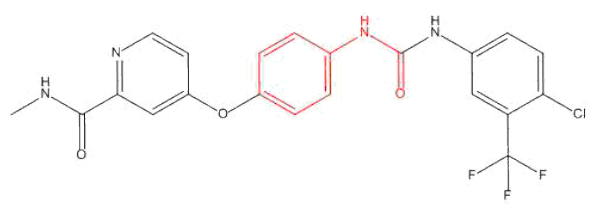

Nexavar $^{\circledR} \mathbf{2}$

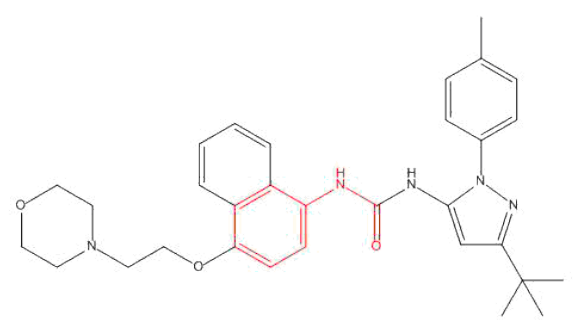

BIRB-796 3

Figure 2: Gleevec ${ }^{\circledast}$, Nexavar ${ }^{\circledR}$ and BIRB-796 highlighting in red the common substructure used during the docking procedure

\section{Rescoring \\ Minimization}

The six poses showing best interaction energy according to $\mathrm{MOE}$, among the 20 obtained from the docking for each ligand-protein set, having also a root mean square deviation (rmsd) between them higher than $0.05 \AA$, were subsequently subjected to a minimization following the already explained protocol.

\section{Binding free energy calculation}

Binding free energies $\left(\Delta G_{\text {bind }}\right)$ were calculated using the MMPB(GB)SA algorithms implemented in the MMPBSA.py program [42]. Thus, the binding free energy is computed as the difference:

$$
\Delta G_{\text {bind }}=\Delta G_{\text {complex }}-\left(\Delta G_{\text {protein }}+\Delta G_{\text {ligand }}\right)
$$

and each term can be estimated as follows:

$$
\Delta G=\Delta G^{0}+\Delta G_{\text {sol }}=\Delta H_{M M}^{0}-T \Delta S^{0}+\Delta G_{\text {sol }}
$$

with the 0 superscript referring to values in vacuo, being $\Delta H_{M M}^{0}$ the molecular mechanics free energy, $\Delta G_{s o l}$ the solvation free energy, and $T \Delta S^{0}$ the entropic contribution. The molecular mechanics energy is in turn calculated as a sum of the internal, electrostatic and van der Waals interactions:

$$
\Delta H_{M M}^{0}=\Delta H_{i n t}^{0}+\Delta H_{e l e}^{0}+\Delta H_{v d w}^{0}
$$

while the solvation free energy is obtained from the polar and nonpolar contributions:

$$
\Delta G_{\text {sol }}=\Delta G_{\text {ele }, \text { sol }}+\Delta G_{\text {nonpol,sol }}
$$

The polar contribution to solvation free energy can be calculated by solving the Poisson-Boltzmann (PB) equation [43] in the case of MMPBSA, or by using the Generalized Born (GB) approach [44] for MMGBSA. 
Finally, the nonpolar contribution to solvation free energy is determined through the solvent accessible area (SASA, $\AA^{2}$ ) according to:

$$
\Delta G_{\text {nonpol,sol }}=\gamma S A S A+b
$$

where $\gamma$ stands for the surface tension $\left(0.0378 \mathrm{kcal} \mathrm{mol}^{-1} \AA^{-2}\right)$ and $b$ is a constant $\left(-0.5692 \mathrm{kcal} \mathrm{mol}^{-1}\right)$.

The entropic term was calculated using normal-mode analysis with default values using the MMPBSA.py program [42]. Taking into account the high computational cost of this approach and the high number of systems to be evaluated this calculation was only performed in the case of the 1-snapshot approach (see below).

In order to evaluate the performance of different MMPB(GB)SA approaches we have explored a set of options. First, we ran MMPBSA and MMGBSA on each of the minimized poses and ranked them according to MMPBSA with $\varepsilon_{\text {int }}=1$. The three best poses were then subjected to a $2 \mathrm{~ns} M D$ run from which we obtained $\Delta \mathrm{G}_{\text {bind }}$ values for the first $1 \mathrm{~ns}$ and the whole trajectory using MMPBSA $\left(\varepsilon_{\text {int }}=1\right)$. Second, we evaluated the influence of $\varepsilon_{\text {int }}$ on the 1-snapshot approach running MMPBSA with $\varepsilon_{\text {int }}$ values of 0.8, 1 and 2. Finally, we evaluated the influence of the parameters used for the Onufriev, Bashford, and Case $G B$ formulation [44] using options $G B=2$ and $G B=5$. Binding free energy decomposition analysis was performed in all cases with option $\mathrm{GB}=2$.

\section{Results \& discussion}

Comparison of methods

Three different criteria were used to evaluate the performance of the methodological approaches compared in this study. First, the percentage of success $(\% S)$ is defined as:

$$
\% S=\frac{T P+T N}{T P+T N+F P+F N}
$$

where TP, TN, FP and FN stand for true positives, true negatives, false positives and false negatives, respectively. According to the experimental IC50 values determined by J. Dietrich et al. [10] we can consider compounds with an IC50 $>10^{4} \mathrm{nM}$ as weak binders. As in all cases there are four weak binders, we have determined TP, TN, FP and FN for each set considering theoretical weak binders those four compounds with worst calculated $\Delta \mathrm{G}_{\text {bind. }}$.

Second, Spearman's rank correlation coefficient, $S p$, which compares the position of each compound when ranked by calculated binding energy to its position when ranked by its experimental IC50 value [45]:

$$
S p=1-\frac{6 \sum d_{i}^{2}}{n\left(n^{2}-1\right)}
$$

being $d_{i}$ the difference in rank for the $i^{\text {th }}$ compound under the two different criteria.

All these parameters, together with the corresponding Pearson's correlation coefficient between $\log (\mathrm{IC} 50)$ and $\Delta \mathrm{G}_{\text {bind }}$, $r$, are presented for all essayed methods on the 3 different proteins ( 3 structures in the case of p38 $\alpha$ ) in Tables 1 and $1 \mathrm{~S}$. 
Table 1 Scoring parameters obtained for the different methodologies applied to the complexes with B-Raf, c-Abl, p38 $\alpha$ Gleevec $^{\circledast}$ (protein structure from the PDB code $3 \mathrm{HEC}$ ), p38 $\alpha$ Nexavar ${ }^{\circledast}$ (protein structure from the PDB code $3 \mathrm{HEG)}$ and p38 $\alpha$ BIRB-796 (protein structure from the PDB code 1KV2). $S$ stands for the $-T \Delta S$ term.

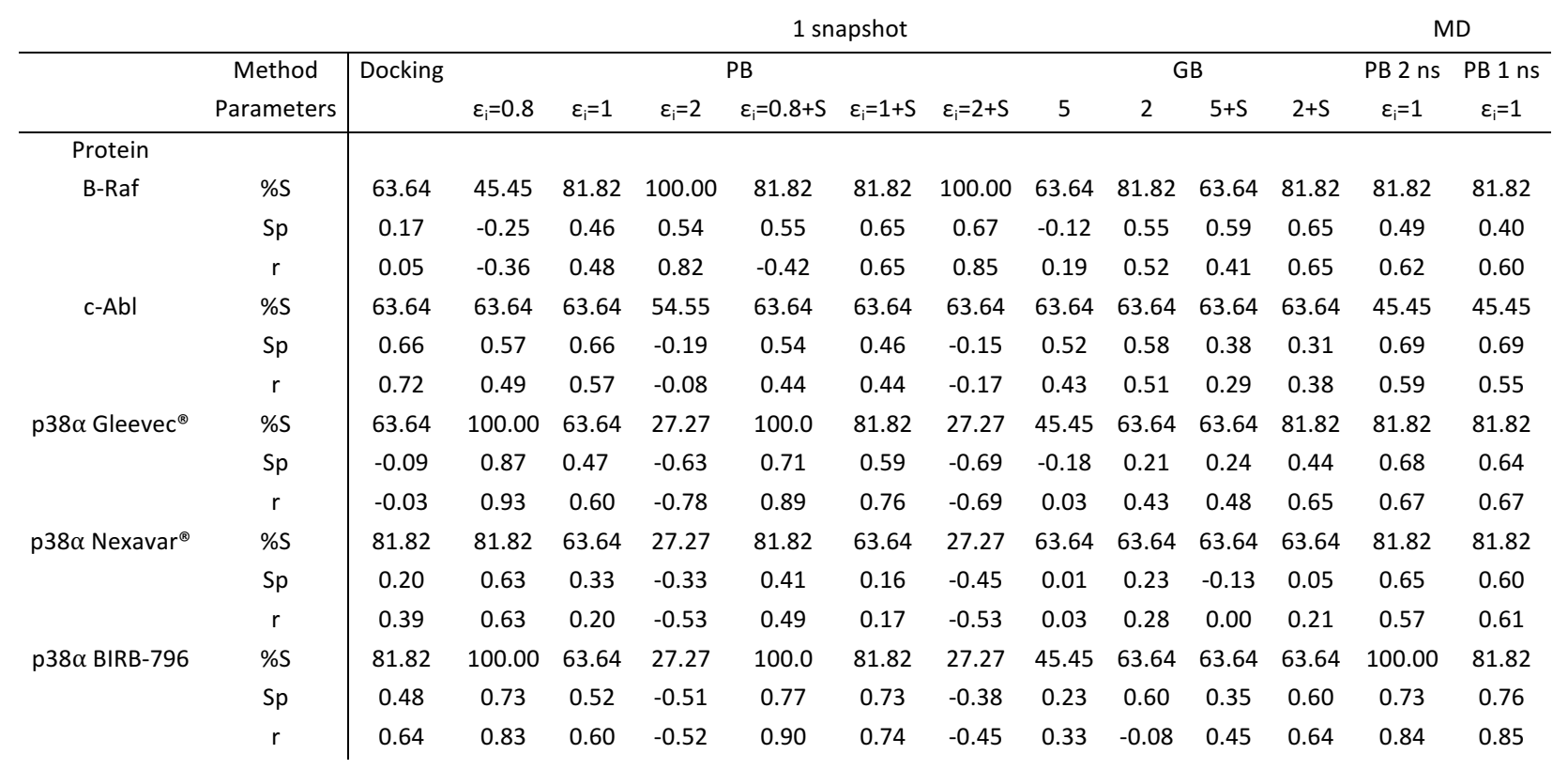

Some conclusions can be extracted from these data. First, comparison of the scoring parameters for $1 \mathrm{~ns}$ and 2 ns MD runs allow to suggest that these calculations are converged. Second, the MD runs provide better results with few exceptions, as can be concluded when comparing column PB $\varepsilon_{\mathrm{i}}=1$, one snapshot, with column for PB $2 \mathrm{~ns}, \varepsilon_{\mathrm{i}}=1$, in Table 1. Thus, MD results are better for all the p38 $\alpha$ complexes, almost the same for B-Raf and slightly worse for c-Abl, as compared to using just one snapshot. Third, there is consensus in the fact that $G B=2$ performs better than $G B=5$, and in the fact that the effect of adding the entropic term is slightly positive for the B-Raf and p38 $\alpha$ sets and almost negligible for the c-Abl set.

In general, post-processing results are better than the docking ones. In the case of B-Raf all post-processing methods, except $\mathrm{GB}=5$, are better than docking; for $\mathrm{c}-\mathrm{Abl}$ none of the post-processing methods improve the docking results, while for $p 38 \alpha$ results are improved in general when $P B$ is used along with $\varepsilon_{i}=0.8$ or 1 (one snapshot) and the MD runs.

Influence of $\varepsilon_{\mathrm{i}}$

The analysis of the influence of the interior dielectric constant in MMPBSA results is a recurrent topic in literature. This interior dielectric constant represents the effect of the protein environment and results are very sensitive to the value used, although the factors that determine its optimal value are not obvious at all [21]. It seems, however, to be a clear tendency to conclude that values higher than the default one $\left(\varepsilon_{i}=1.0\right)$ are expected to improve results [46-48]. Moreover, Wang et al. [49], after analysing 54 papers on MMPBSA and MMGBSA free energy calculations, concluded as a general guideline that an $\varepsilon_{\mathrm{i}}=1$ should be used for non-polar binding sites, $\varepsilon_{\mathrm{i}}=2.0$ for polar binding sites, and $\varepsilon_{\mathrm{i}}=4$ when the binding site is composed of charged residues. In this work, we have essayed an interior dielectric constant higher than the default one $\left(\varepsilon_{\mathrm{i}}=2.0\right)$. Besides, considering $\varepsilon_{\mathrm{i}}$ just as an adjustable parameter we additionally checked the influence of a lower value $\left(\varepsilon_{\mathrm{i}}=0.8\right)$.

Table 1 allows to see that the influence of the $\varepsilon_{\mathrm{i}}$ value depends on the set studied. Thus, results for the B-Raf set improve when $\varepsilon_{\mathrm{i}}$ increases, reaching a $100 \%$ of success and a Pearson's correlation coefficient of 0.85 for $\varepsilon_{\mathrm{i}}=2+\mathrm{S}$. In the case of the p38 $\alpha$ Gleevec $^{\circledR}$ set $\%$ S improves from $27.3 \%$ with $\varepsilon_{i}=2.0$ to $100 \%$ with $\varepsilon_{i}=0.8$, all remaining scoring parameters following the same trend for all three $p 38 \alpha$ sets. Finally, c-Abl scoring parameters show a small dependence with the $\varepsilon_{\mathrm{i}}$ value essayed. 
In order to get a deeper insight into the influence of $\varepsilon_{\mathrm{i}}$ on the calculated results Table 2 renders the change in ranking position of each of the 11 studied ligands when the interior dielectric constant increases, along with the corresponding experimental ranking [10]. In the case of B-Raf, experimental IC50 values show that positively charged ligands $(1,4,5$ and 10) bind weakly, which is not predicted with $\varepsilon_{\mathrm{i}}=0.8$, being compound one predicted as the best one when using this interior dielectric constant value. Interestingly, when $\varepsilon_{\mathrm{i}}$ increases the position in the ranking for ligands 1 and 4 sharply worsens and they are correctly ranked as weak binders for $\varepsilon_{i}=2.0$. The position in the ranking of non-charged ligands does not show a clear tendency. Thus, as a consequence of the influence of $\varepsilon_{i}$ for charged ligands the global result is that the ranking coefficients improve when $\varepsilon_{\mathrm{i}}$ increases.

Positively charged ligands are also predicted to bind weakly to p38 $\alpha$, and inversely to what was seen for B-Raf, a decrease in $\varepsilon_{i}$ worsens the ranking position of charged ligands thus increasing all ranking coefficients from $\varepsilon_{i}=2.0$ to $\varepsilon_{\mathrm{i}}=0.8$. Moreover, all four inactive compounds are correctly ranked as weak binders for $\varepsilon_{\mathrm{i}}=0.8$, except in the p38 $\alpha$ Nexavar ${ }^{\circledast}$ case, for which compound 11 is predicted to be inactive instead of compound 10.

$\mathrm{c}-\mathrm{Abl}$, on the other hand, is the only protein to which Gleevec ${ }^{\circledR}$, positively charged, binds strongly, while ligands 4 and 5 (positively charged), and ligands 3 and 7 (with no charge) are predicted to bind weakly according to the IC50 values [10]. The behaviour of charged ligands is the same as for $p 38 \alpha$, with ligands $1,4,5$ and 10 being the best ranked if $\varepsilon_{i}=2$ is used, and the worst ranked when $\varepsilon_{i}=0.8$ is used. The fact that neutral compounds do not follow a clear trend, together with the fact that ligand 1, which binds strongly, follows the same trend than weak binders, explain why the ranking coefficients for c-Abl do not correlate clearly with $\varepsilon_{i}$. They also explain why the c-Abl set results are worse than those for the other sets.

Table 2: Evolution of the ranking in binding affinity (from highest to lowest) with $\varepsilon_{\mathrm{i}}$ for one-snapshot methods with no entropy term. Horizontal line separates strong binders from weak binders.

\begin{tabular}{|c|c|c|c|c|c|c|c|c|c|c|c|c|c|c|c|c|c|}
\hline \multicolumn{4}{|c|}{ B-Raf } & \multicolumn{4}{|c|}{ c-Abl } & \multicolumn{4}{|c|}{ p38 $\alpha$ Gleevec $^{\circledR}$} & \multicolumn{3}{|c|}{ p38 $\alpha$ Nexavar $^{\oplus}$} & \multicolumn{3}{|c|}{ p38 $\alpha$ BIRB-796 } \\
\hline Experimental & & $\varepsilon_{\mathrm{i}}$ & & Experimental & & $\varepsilon_{\mathrm{i}}$ & & Experimental & & & & & $\varepsilon_{\mathrm{i}}$ & & & & \\
\hline Ligand & 0.8 & 1.0 & 2.0 & Ligand & 0.8 & 1.0 & 2.0 & Ligand & 0.8 & 1.0 & 2.0 & 0.8 & 1.0 & 2.0 & 0.8 & 1.0 & 2.0 \\
\hline 7 & 1 & 8 & 8 & 6 & 8 & 8 & 1 & 7 & 3 & 3 & 10 & 9 & 9 & 10 & 3 & 3 & 10 \\
\hline 2 & 8 & 3 & 3 & 8 & 3 & 3 & 4 & 9 & 9 & 9 & 1 & 3 & 3 & 9 & 8 & 8 & 3 \\
\hline 3 & 3 & 9 & 9 & 1 & 6 & 6 & 5 & 3 & 6 & 8 & 4 & 7 & 10 & 8 & 7 & 7 & 5 \\
\hline 11 & 10 & 7 & 6 & 9 & 9 & 9 & 10 & 6 & 7 & 6 & 5 & 8 & 8 & 5 & 9 & 9 & 1 \\
\hline 6 & 9 & 6 & 7 & 2 & 7 & 1 & 8 & 2 & 8 & 10 & 3 & 10 & 7 & 3 & 6 & 6 & 4 \\
\hline 9 & 4 & 2 & 2 & 11 & 2 & 7 & 3 & 11 & 2 & 1 & 9 & 6 & 5 & 4 & 11 & 10 & 8 \\
\hline 8 & 7 & 1 & 11 & 10 & 11 & 2 & 6 & 8 & 11 & 7 & 8 & 2 & 2 & 1 & 2 & 5 & 9 \\
\hline 1 & 6 & 10 & 10 & 7 & 1 & 11 & 9 & 1 & 1 & 2 & 6 & 5 & 6 & 7 & 10 & 11 & 7 \\
\hline 4 & 2 & 4 & 4 & 3 & 10 & 10 & 7 & 4 & 10 & 11 & 7 & 11 & 1 & 2 & 5 & 1 & 6 \\
\hline 5 & 5 & 11 & 1 & 4 & 4 & 4 & 2 & 5 & 5 & 4 & 2 & 4 & 11 & 6 & 1 & 2 & 2 \\
\hline 10 & 11 & 5 & 5 & 5 & 5 & 5 & 11 & 10 & 4 & 5 & 11 & 1 & 4 & 11 & 4 & 4 & 11 \\
\hline
\end{tabular}

In order to have a closer look, Table $2 \mathrm{~S}$ collects $\Delta \mathrm{H}_{\text {ele }}^{0}+\Delta \mathrm{G}_{\text {ele,sol }}$ from the MMPBSA calculation, in the one-snapshot approximation with no entropic term, for each of the proteins and a representative neutral and charged ligand. Thus, for B-Raf we can compare Gleevec ${ }^{\circledR}$ with Nexavar ${ }^{\circledR}$ and see that the $\Delta H_{\text {ele }}^{0}+\Delta G_{\text {ele,sol }}$ term follows opposite trends for Gleevec $^{\circledast}$ or Nexavar ${ }^{\circledast}$, increasing its value with $\varepsilon_{i}$ for the first, while decreasing it for the latter, which correlates with the change in ranking positions seen in Table 2 . The opposite is seen for $p 38 \alpha$ Gleevec $^{\circledR}$, while c-Abl shows the same trend for charged or neutral ligands. 
With all these results in mind it can now be decided which of all the essayed methods is able to reproduce best the experimental results and thus is supposed to render the most trustable structures. Our protocol chooses the best onesnapshot method and compare its results with those from the classical MD approach for each of the studied sets, on the basis of the scoring parameters shown in Table 1. Hence, in all cases we compare one option using just one snapshot, with the classical option of using a MD run. One-snapshot MMPBSA methods were chosen in all cases, with $\varepsilon_{\mathrm{i}}=2+\mathrm{S}$ for B-Raf, $\varepsilon_{\mathrm{i}}=1$ for C-Abl, $\varepsilon_{\mathrm{i}}=0.8$ for p $38 \alpha$ Gleevec $^{\circledR}, \varepsilon_{\mathrm{i}}=0.8$ for p38 $\alpha$ Nexavar $^{\circledR}$ and $\varepsilon_{\mathrm{i}}=0.8+\mathrm{S}$ for p38 $\alpha$ BIRB796. All of them yielded higher or similar values of the scoring parameters of Table 1 than the remaining one-snapshot methods. Table 3 compares the experimental ranking of those ligands considered strong binders (IC50 < $\left.10^{4} \mathrm{nM}\right)$ with the predicted rankings from the best one-snapshot method and with the predicted raking from the 2 ns MD MMPBSA calculation. Besides, Table 3S renders the same comparison for those ligands considered weak binders. Both tables also show which pose is predicted to exhibit the best $\Delta G_{\text {bind }}$ for each of the MMPBSA options compared in each case.

As concerns the B-Raf set, the three scoring parameters are better for the one-snapshot method with $\varepsilon_{\mathrm{i}}=2+\mathrm{S}$ than for 2 ns MD. We will later analyse ligand 2 (for which there is an experimental structure) and ligand 7 (the best ligand for B-Raf according to the experimental results). The binding poses predicted for these two ligands are the same for the best one-snapshot and MD method (see Table 3, pose 5 for ligand 7 and pose 4 for ligand 2). Considering that both approaches predict the same ligand conformations (poses) to be analysed, it is better to use the MD run, as it will allow to have information about the strength of established $\mathrm{H}$-bonds. Thus, for this set we will analyse the structures from a snapshot extracted after 1 ns of MD.

In the case of c-Abl, the one-snapshot method with $\varepsilon_{i}=1.0$ and no entropic term exhibits slightly better \%S and $r$ values, although worse Sp, than MMPBSA with 2 ns of MD. The interactions established by c-Abl with ligands 1 (for which there is an experimental structure) and 6 (the best ligand for c-Abl) will later be analysed. Taking into account the similar values obtained for the scoring parameters of Table 1 with both methods, together with the fact that the ranking of ligand 6 is better reproduced and no experimental structure is available for ligand 6, we decided to analyse the structure of pose 1 for ligand 1 and pose 2 for ligand 6 with a snapshot extracted after 1 ns of MD (see Table 3).

Table 3: Comparison between the experimental ranking for strong binders (IC50<10 $\mathrm{nM}$ ) and the rankings predicted from the best one-snapshot method or from 2 ns of MD (average values, standard error in parenthesis). Ligands and their corresponding pose whose interactions are analysed later are shown in bold. Energies in kcal mol ${ }^{-1}$. $\mathrm{S} \mathrm{stands} \mathrm{for}$ the $\mathrm{T} \Delta \mathrm{S}$ term

\begin{tabular}{|c|c|c|c|c|c|c|c|c|}
\hline \multirow[b]{2}{*}{ Protein } & \multicolumn{2}{|c|}{ Experimental } & \multicolumn{3}{|c|}{ One snapshot } & \multicolumn{3}{|c|}{ MD } \\
\hline & Ligand & IC50 (nM) & Ligand & Pose & $\Delta G_{\text {bind }}$ & Ligand & Pose & $\Delta \mathrm{G}_{\text {bind }}$ \\
\hline \multirow[t]{8}{*}{ B-Raf } & & & & & $\mathrm{PB} \varepsilon_{\mathrm{i}}=2+\mathrm{S}$ & & & $\mathrm{PB} \varepsilon_{\mathrm{i}}=1$ \\
\hline & 7 & $<1$ & 8 & 5 & -138.5 & 8 & 2 & $-112.5(0.3)$ \\
\hline & 2 & 76.2 & 3 & 1 & -136.3 & 9 & 4 & $-104.7(0.4)$ \\
\hline & 3 & 83.4 & 9 & 4 & -133.8 & 7 & 5 & $-100.3(0.3)$ \\
\hline & 11 & 92.3 & 6 & 5 & -124.0 & 6 & 5 & $-97.7(0.3)$ \\
\hline & 6 & 180.1 & 7 & 5 & -123.1 & 3 & 1 & $-96.6(0.4)$ \\
\hline & 9 & 236.7 & 2 & 4 & -122.1 & 2 & 4 & $-94.3(0.2)$ \\
\hline & 8 & 413.9 & 11 & 5 & -112.8 & 10 & 4 & $-91.7(0.7)$ \\
\hline \multirow[t]{8}{*}{ c-Abl } & & & & & PB $\varepsilon_{\mathrm{i}}=1.0$ & & & \\
\hline & 6 & $<1$ & 8 & 2 & -113.32 & 8 & 2 & $-114.7(0.3)$ \\
\hline & 8 & 8.6 & 3 & 1 & -107.12 & 6 & 2 & $-105.0(0.3)$ \\
\hline & 1 & 10.8 & 6 & 3 & -101.77 & 3 & 2 & $-102.8(0.4)$ \\
\hline & 9 & 62.6 & 9 & 9 & -99.91 & 9 & 9 & $-100.4(0.3)$ \\
\hline & 2 & 225.9 & 1 & 7 & -98.45 & 7 & 2 & $-97.7(0.3)$ \\
\hline & 11 & 244 & 7 & 2 & -92.79 & 1 & 1 & $-95.8(0.3)$ \\
\hline & 10 & 572.4 & 2 & 2 & -87.05 & 2 & 2 & $-88.1(0.3)$ \\
\hline \multirow[t]{3}{*}{ p38 $\alpha$ Gleevec ${ }^{\circledR}$} & & & & & PB $\varepsilon_{\mathrm{i}}=0.8$ & & & \\
\hline & 7 & $<1$ & 3 & 5 & -91.1 & 3 & 6 & $-108.0(0.4)$ \\
\hline & 9 & $<1$ & 9 & 1 & -83.6 & 9 & 8 & $-102.5(0.3)$ \\
\hline
\end{tabular}




\begin{tabular}{|c|c|c|c|c|c|c|c|c|}
\hline & 3 & 4 & 6 & 7 & -78.5 & 8 & 18 & $-101.7(0.3)$ \\
\hline & 6 & 18.4 & 7 & 3 & -77.8 & 6 & 7 & $-99.8(0.3)$ \\
\hline & 2 & 84.8 & 8 & 18 & -77.0 & 1 & 11 & $-95.0(0.4)$ \\
\hline & 11 & 105.5 & 2 & 4 & -71.1 & 7 & 3 & $-92.5(0.2)$ \\
\hline & 8 & 189.3 & 11 & 16 & -68.9 & 2 & 4 & $-84.0(0.3)$ \\
\hline \multirow[t]{8}{*}{ p38 $\alpha$ Nexavar ${ }^{\circledast}$} & & & & & PB $\varepsilon_{\mathrm{i}}=0$. & & & \\
\hline & 7 & $<1$ & 9 & 9 & -76.6 & 3 & 5 & $-102.7(0.4)$ \\
\hline & 9 & $<1$ & 3 & 5 & -75.7 & 8 & 2 & $-98.6(0.4)$ \\
\hline & 3 & 4 & 7 & 5 & -69.8 & 9 & 14 & $-95.3(0.3)$ \\
\hline & 6 & 18.4 & 8 & 16 & -68.9 & 7 & 5 & $-87.2(0.4)$ \\
\hline & 2 & 84.8 & 10 & 19 & -65.6 & 6 & 20 & $-84.9(0.3)$ \\
\hline & 11 & 105.5 & 6 & 18 & -65.1 & 5 & 2 & $-84.2(0.4)$ \\
\hline & 8 & 189.3 & 2 & 15 & -64.3 & 2 & 15 & $-80.0(0.3)$ \\
\hline \multirow[t]{8}{*}{ p38 $\alpha$ BIRB-796 } & & & & & $B \varepsilon_{i}=0.8$ & & & \\
\hline & 7 & $<1$ & 3 & 8 & -90.2 & 8 & 6 & $-106.1(0.6)$ \\
\hline & 9 & $<1$ & 7 & 3 & -82.5 & 3 & 8 & $-104.8(0.3)$ \\
\hline & 3 & 4 & 8 & 20 & -75.1 & 7 & 3 & $-93.9(0.2)$ \\
\hline & 6 & 18.4 & 6 & 13 & -70.6 & 6 & 4 & $-93.4(0.3)$ \\
\hline & 2 & 84.8 & 11 & 10 & -69.1 & 9 & 9 & $-91.6(0.3)$ \\
\hline & 11 & 105.5 & 9 & 18 & -66.5 & 2 & 8 & $-86.4(0.3)$ \\
\hline & 8 & 189.3 & 2 & 3 & -58.4 & 11 & 14 & $-86.3(0.2)$ \\
\hline
\end{tabular}

The $\mathrm{p} 38 \alpha$-Gleevec ${ }^{\circledR}$ complex is interesting because the experimental structure describes a structure with a ligand that is expected to bind weakly to the protein (IC50 $>10^{4} \mathrm{nM}$ ). Results from the best one-snapshot method are clearly better than those from MMPBSA with $2 \mathrm{~ns}$ of MD and the latter also predicts ligand 1 to bind strongly to the protein, which is not reported to happen according to the IC50 value (see Table 3, ligand 1 pose 11 has a predicted $\Delta G_{\text {bind }}=-95$ $\mathrm{kcal} \mathrm{mol}^{-1}$ ). Consequently, we will analyse the one-snapshot poses for p38 $\alpha$ with Gleevec ${ }^{\circledast}$ (experimental structure) and ligands 7 and 9 (with the lowest IC50 values). These poses are thus (see Tables 3 and 3S) pose 1 for ligand 1, pose 3 for ligand 7, and pose 1 for ligand 9.

In the case of $\mathrm{p} 38 \alpha$ Nexavar $^{\circledR}$ the scoring parameters from the best one-snapshot method and and MMPBSA with 2 ns of $\mathrm{MD}$ are almost identical. We decided to analyse the one-snapshot poses as the relative ranking for the ligands whose interactions were going to be examined later (ligands 1, 2, 7 and 9) matches better with the experimental results (see Tables 3 and 3S). According to this criterion the poses to be analysed were pose 6 of ligand 1, pose 15 of ligand 2, pose 5 of ligand 7 and pose 9 of ligand 9.

Finally, for the p38 $\alpha$ BIRB-796 structure, the comparison between the predicted scoring from the best one-snapshot with that from MMPBSA with 2 ns of MD allow us to choose the poses predicted by the first method, using the same arguments than those for the $\mathrm{p} 38 \alpha \mathrm{Nexavar}^{\circledR}$ set. So, the predicted interactions to be analysed are those established between the protein p38 $\alpha$ BIRB-796 and pose 8 of ligand 3, pose 1 of ligand 1, pose 3 of ligand 7 and pose 18 of ligand 9.

\section{Analysis of the selected predicted poses}

The analysis, from an energetic point of view, will use the different terms arising from the MMGBSA energy decomposition analysis run, as well as information of the different $\mathrm{H}$-bonds established, for each of the methods and poses selected as the most trustable in section 3.3. These data appear in Tables 4S-8S, while Figures 3 and 4 try to focus on highlighting those residues of each protein that interact differently with each ligand (difference interaction spectrum), and on visualizing the polar or non-polar character of those differences, respectively. Yang et al. [50] divided the binding pocket of $\mathrm{p} 38 \alpha$ used by Gleevec ${ }^{\circledR}, \mathrm{Nexavar}^{\circledR}$ and BIRB-796 into a conserved hydrophobic pocket (HP) and non-conserved allosteric pocket (AP), comprised of two sites, and connected with inhibitor's selectivity. In this sense, we have aligned the sequences of the three proteins with UCSF Chimera [31] with the aim of identifying residues in B-Raf and c-Abl equivalent to those composing the HP and AP in p38 $\alpha$ (see Figure 1S). Tables 4S-8S try to clarify the information provided by colouring residues from the HP red and residues from the AP blue. 
The superposition of the experimental structure of B-Raf complexed with Nexavar ${ }^{\circledR}$ [35], with the theoretical conformations adopted by Nexavar ${ }^{\oplus}$ and ligand 7 (Figure 2S), allows concluding that the theoretical pose is very similar to the experimental one, which suggests that the chosen methodology is suitable. Besides, as expected taking into account that the placement stage was avoided during the docking process, the common substructure has maintained its position. 
a)

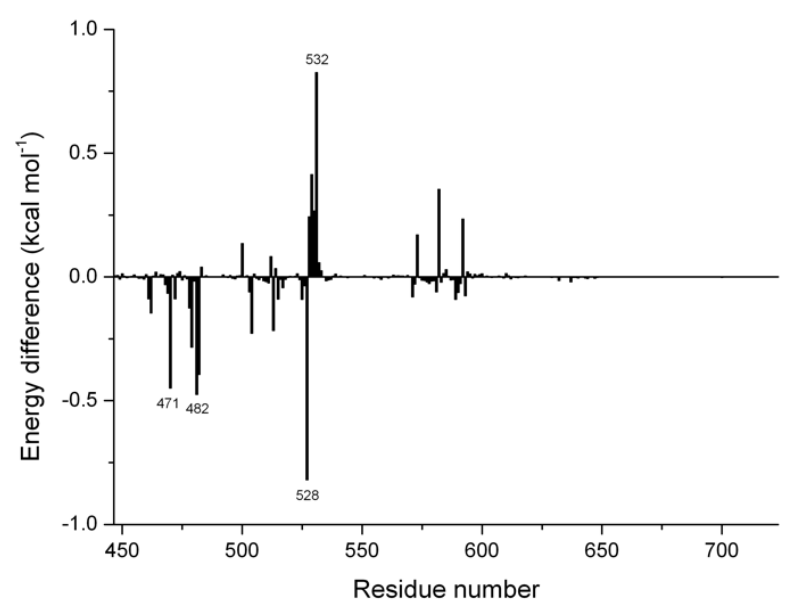

b)

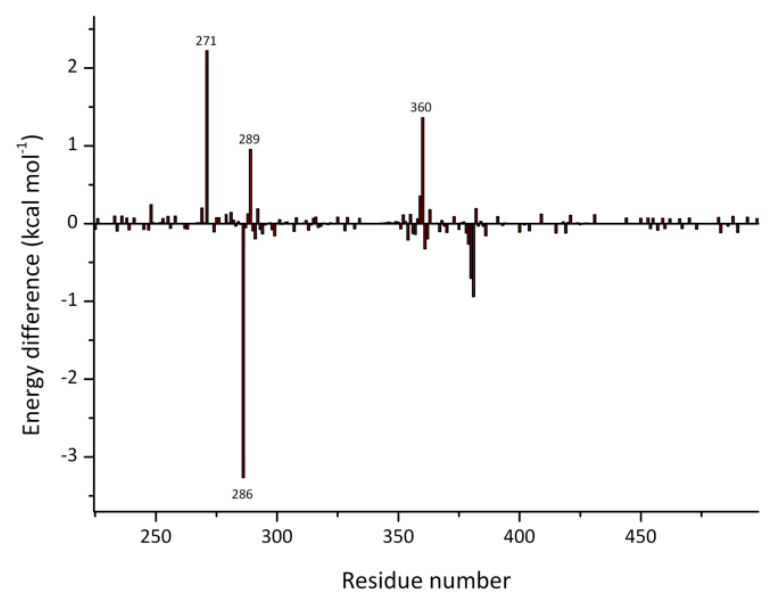

c)

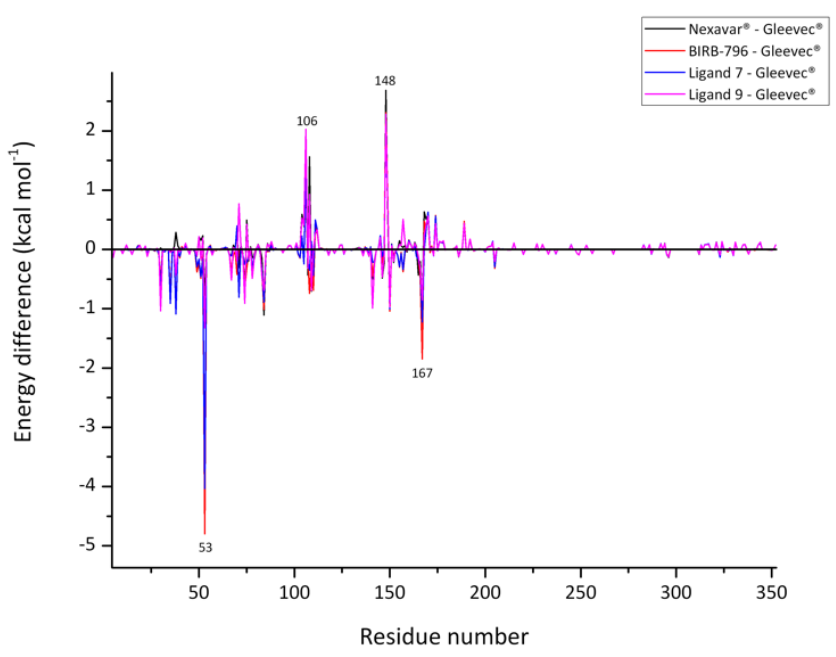

Figure 3: Difference interaction spectrum a) (ligand $7-$ Nexavar $^{\circledR}$ ) for B-Raf, b) (ligand $6-$ Gleevec $^{\circledR}$ ) for c-Abl and c)

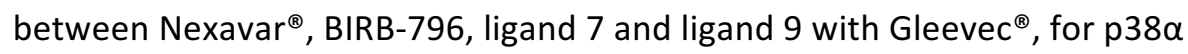


(a)

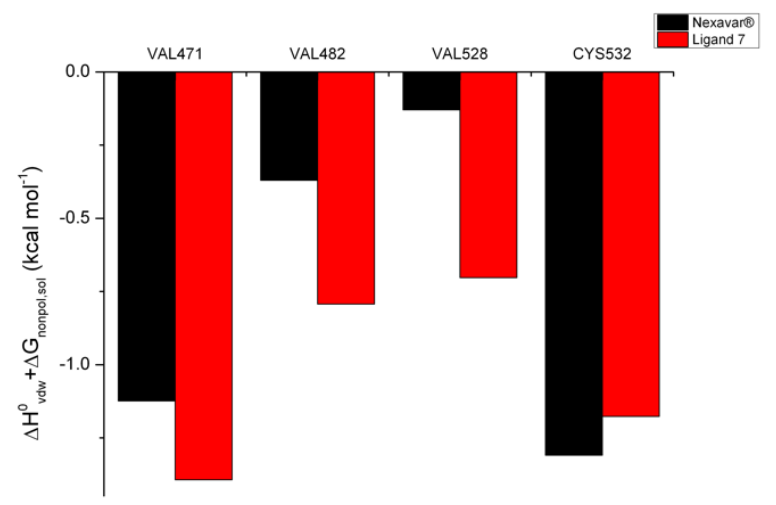

(c)

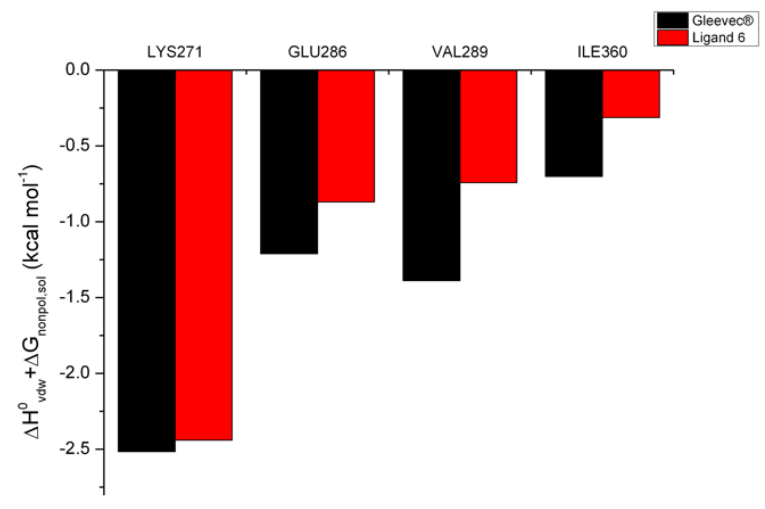

(e)

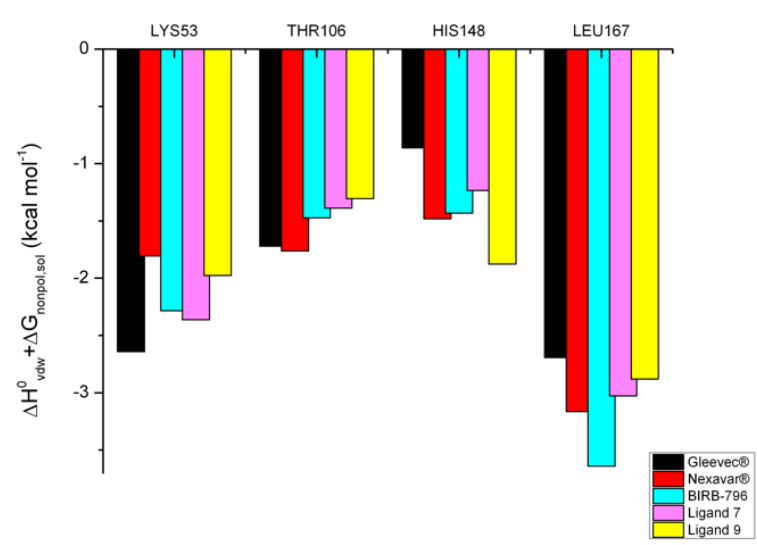

(b)

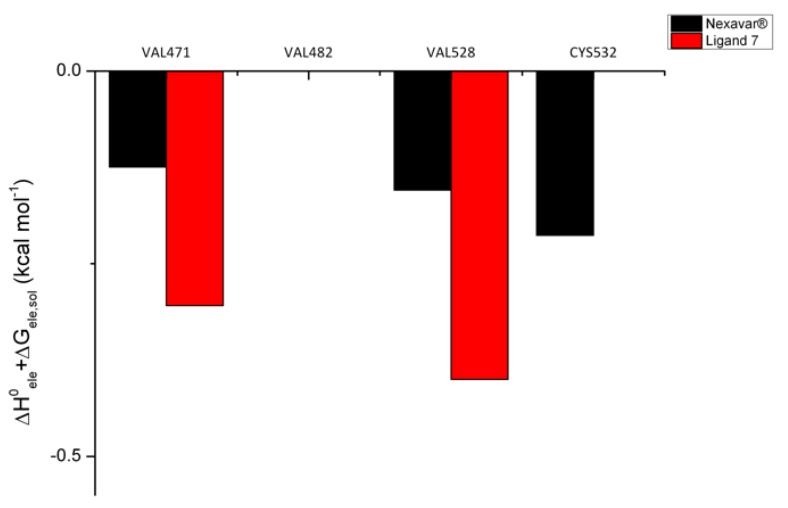

(d)

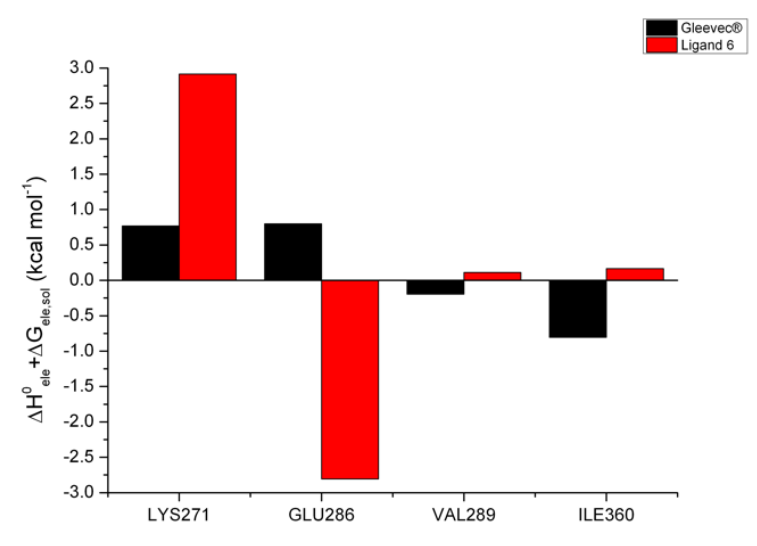

(f)

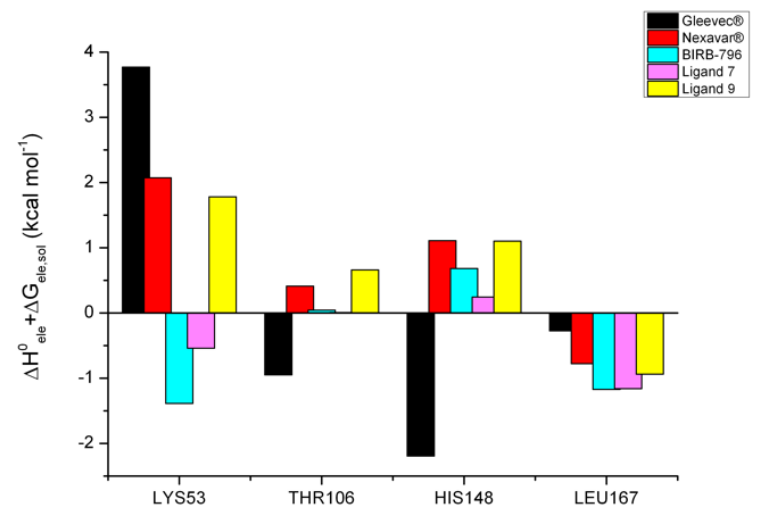

Figure 4: Non-polar and polar contributions to free binding energy for selected residues of B-Raf, C-Abl and p38 $\alpha$ interacting with different ligands. (a) and (b) correspond to non-polar and polar contributions for B-Raf interacting with Nexavar ${ }^{\circledR}$ and ligand 7, respectively. (c) and (d) correspond to non-polar and polar contributions for c-Abl interacting with Gleevec ${ }^{\circledR}$ and ligand 6. (e) and (f) correspond to non-polar and polar contributions for p38 $\alpha$ interacting with Gleevec $^{\circledR}$, Nexavar ${ }^{\circledast}$, BIRB-796, ligand 7 and ligand 9 
Focussing on the comparison between ligands $2($ IC50 $=76.2 \mathrm{nM})$ and $7($ IC50 $<1 \mathrm{nM})$, Figure 5 compares both predicted structures. As seen in Table 4S, B-Raf establishes similar H-bonds with both ligands (through GLU501, CYS532 and ASP594). It is worth to mention the H-bond network established by ASP594...Nexavar ${ }^{\circledR}$...GLU501...LYS483, previously described for DFG-out conformations of B-Raf [35], c-Abl [34, 51] and p38 $\alpha$ [37]. It is thus very interesting to realise, see Figure $3 \mathrm{~S}$, that a small relaxation of the structure allows to increase the $\mathrm{H}$-bond network to include a $\mathrm{H}$ bond between LYS483 and ASP594 for both Nexavar ${ }^{\circledR}$ and ligand 7, with lifetimes of the $100 \%$ of the trajectory for all H-bonds.

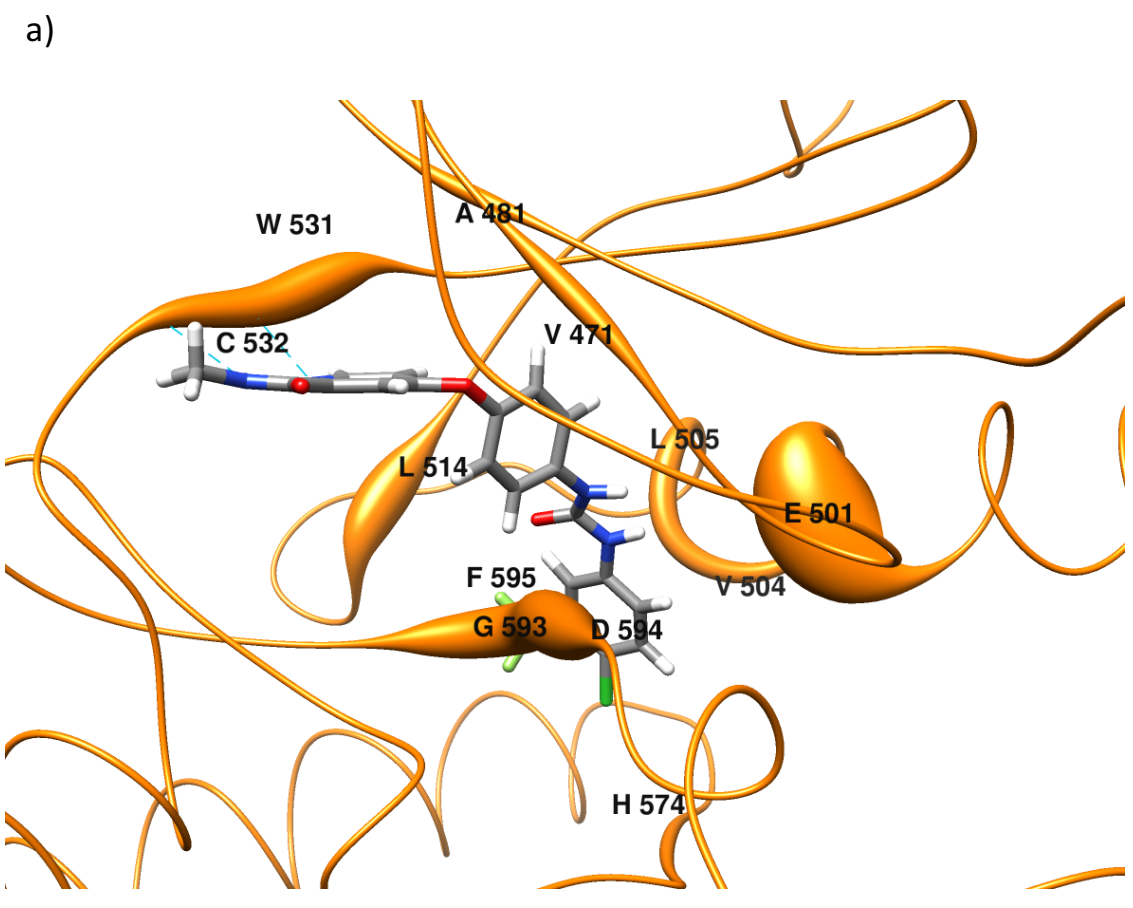

b)

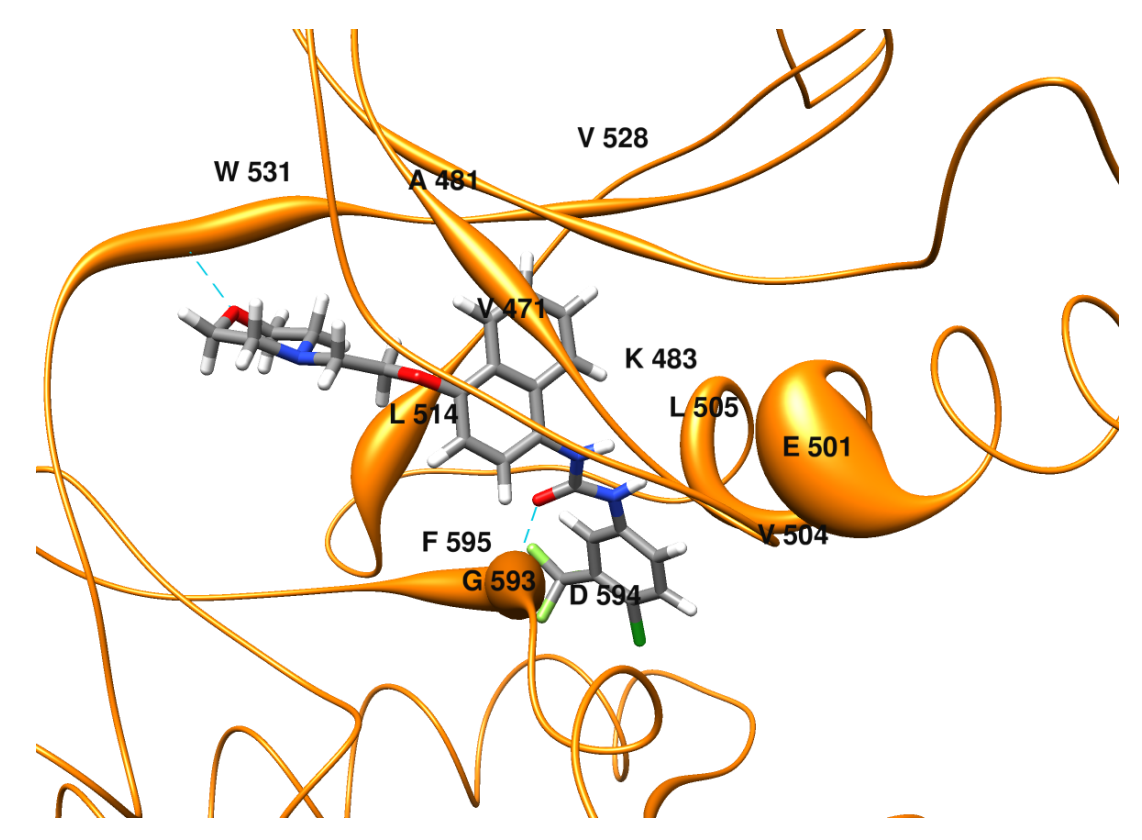

Figure 5: Theoretical structures of a) ligand 2 (Nexavar $\left.{ }^{\circledast}\right)$ and b) ligand 7 docked into B-Raf, showing the protein with worm radii proportional to the MMGBSA predicted contribution of each residue to binding energy.

From a structural point of view, therefore, it seems that the biggest difference between Nexavar ${ }^{\circledR}$ and ligand 7 is the change of benzene with naphthalene in the central core, whose bigger size could help establishing stronger interactions with the hydrophobic pocket. Figure 3 allows concluding on the one hand that the differences between 
both ligands are subtle, and on the other hand that interactions are stronger for ligand 2 in the zone around CYS532 (hinge region), and for ligand 7 for the HP and its surroundings (VAL471, VAL482, VAL528). Besides, Figure 4 shows that the differences for CYS532 are due to the fact that Nexavar ${ }^{\circledR}$ establishes two $\mathrm{H}$-bonds with CYS532, while ligand 7 can only establish one $\mathrm{H}$-bond using the oxygen atom of the morpholine ring. Finally, it becomes apparent that the stronger interactions for residues VAL471, VAL482 and VAL528 are caused by the change from benzene to naphthalene. From this data, we suggest that a ligand with naphthalene in the central core as ligand 7, but with a heteroaromatic moiety instead of the morpholine ring (so that it can establish $\pi-\pi$ interactions with TRP531), would be even more active against ${ }^{{ }^{6} 00 E_{B}} \mathrm{~B}$-Raf. Dietrich et al. [10] already pointed out from the comparison between compounds 7 and 11 that favourable hydrophobic binding interactions were expected when a naphthyl ring is utilized to bind the gatekeeper region of B-Raf.

$c-A b l$

Comparison of the experimental conformation adopted by Gleevec ${ }^{\circledR}$ with the predicted one and that of ligand 6 shows the common substructures to overlap (Figure 4S), while the 1-chloro-2-(trifluoromethyl)benzene ring of ligand 6 occupies a zone different than that occupied by the 1-methyl-piperazine ring of Gleevec ${ }^{\circledR}$.Figure 6 allows to realise that the relative conformation adopted by the 1-chloro-2-(trifluoromethyl)benzene ring in ligand 6 is precisely that adopted by Nexavar ${ }^{\circledast}$ when bound to B-Raf. Also, as previously described for B-Raf, a H-bond network formed by ASP381...LIGAND...GLU286...LYS271...ASP381 is seen throughout the trajectory (lifetimes of more than $94 \%$, distances $\sim 3 \AA$ ) for both Gleevec ${ }^{\circledR}$ and ligand 6 . It is worth to emphasize that the recently discovered c-Abl inhibitor CHMFL-074, whose X-ray structure was solved with $1.53 \AA$ of resolution (PDB code $5 \mathrm{HU}$ [52], experimental IC50 = $24 \mathrm{nM}$ ) also displays the extended $\mathrm{H}$-bond interaction, even improved with an additional $\mathrm{H}$-bond from LYS271 to GLY383. The superposition of the conformations adopted by the ligands docked into c-Abl studied in the present work with that of CHMFL-074 (Figure 4S) shows how the new inhibitor includes both the 1-methyl-piperazine and the 1-chloro-2(trifluoromethyl)benzene rings, each ring located in the same place as the corresponding one for Gleevec ${ }^{\circledR}$ and ligand 6 , respectively. The structural analysis from Figure 6 highlights the fact that having a urea linker (ligand 6) instead of an acetamide (Gleevec ${ }^{\circledR}$ ) allows establishing $2 \mathrm{H}$-bonds instead of one between the ligand and GLU286.

Besides, interactions established through the 1-methyl-piperazine ring explain the better values predicted from the energy decomposition analysis for residues VAL289 and ILE360 in the case of Gleevec ${ }^{\circledast}$ (Figure 3). The importance of interactions between the 1-methyl-piperazine ring and ILE360, HIS361 and PHE359 was highlighted previously [10]. Our calculations allow to quantify their importance. Thus, the H-bond described with ILE360 has a lifetime of $74 \%$ of the trajectory, while that with HIS361 is not predicted to be stable. PHE359 establishes indeed better interactions with the 1-methyl-piperazine ring (Figure 3), but the difference is almost negligible. Our results strongly suggest that the key difference between ligand 6 and Gleevec ${ }^{\circledR}$ is the interaction with GLU286, being that difference almost exclusively of polar character (Figure 4). Hence, from this data we suggest that a structural modification of Gleevec ${ }^{\circledR}$ or CHMFL074 changing the acetamide linker with a urea type one would improve their c-Abl inhibition. The possibility of using a urea type linker was essayed by Dietrich et al. [10], who tried to improve compound 4 (IC50 > $30000 \mathrm{nM}$ ) with the urea linker (compound 11, IC50 $=244.0 \mathrm{nM}$ ). 
a)

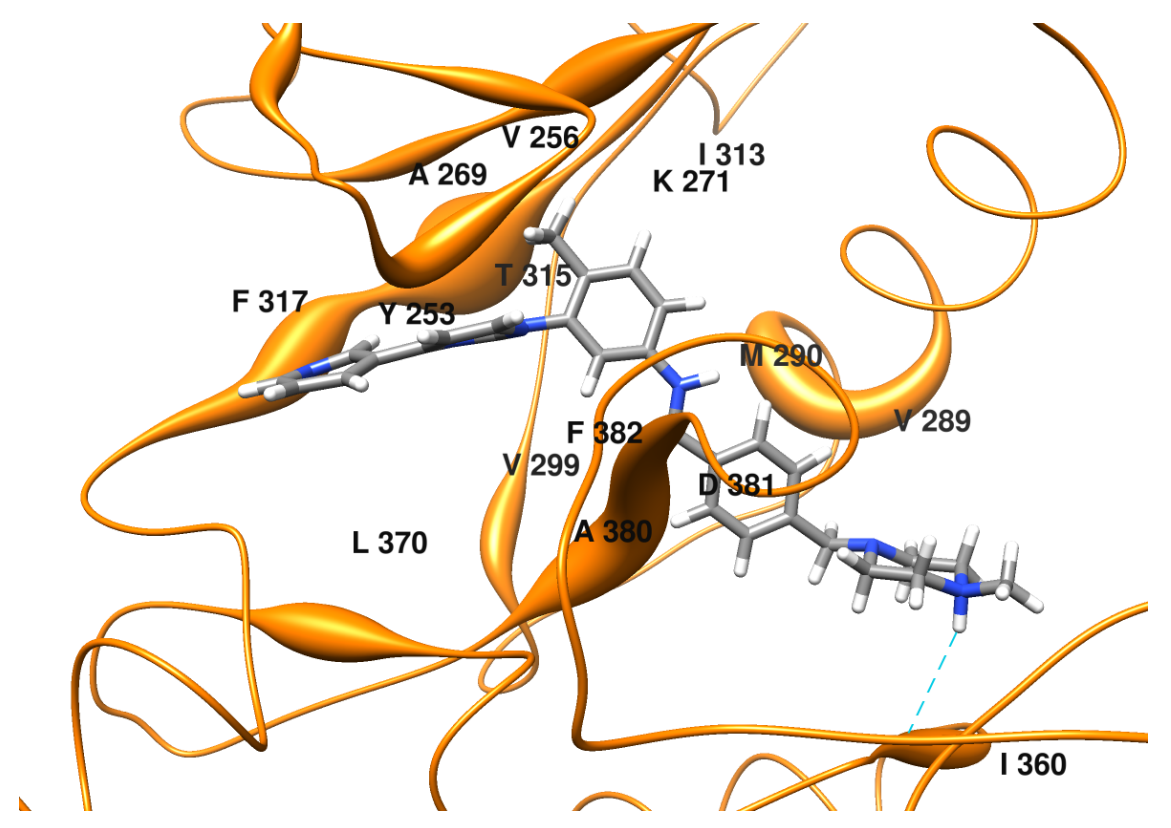

b)

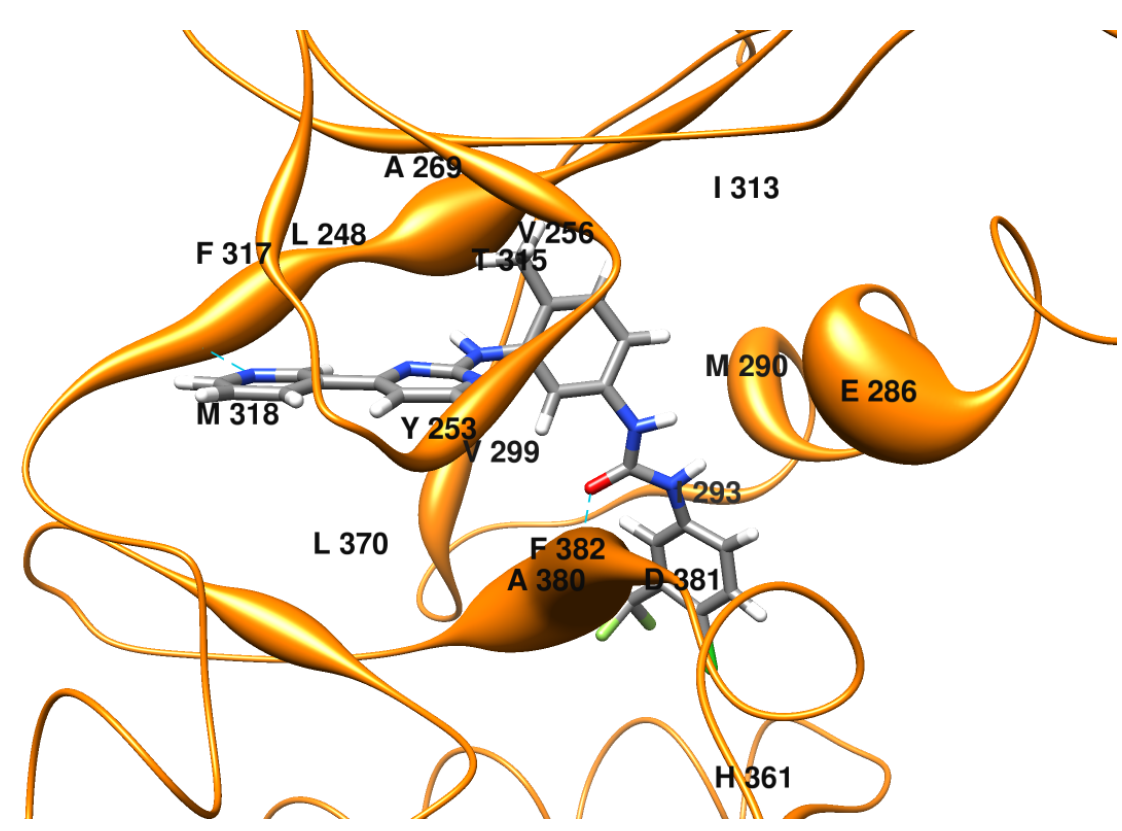

Figure 6: Theoretical structures of a) ligand $1\left(\right.$ Nexavar $\left.^{\circledR}\right)$ and b) ligand 6 docked into c-Abl, showing the protein with worm radii proportional to the MMGBSA predicted contribution of each residue to binding energy. 
The analysis of the p38 $\alpha$ sets now implies not only the predicted conformations found for Gleevec ${ }^{\circledR}\left(I C 50>10^{4} \mathrm{nM}\right)$, Nexavar ${ }^{\circledast}($ IC50 $=84.8 \mathrm{nM})$, BIRB-796 $($ IC50=4.0 nM), ligand $7($ IC50 $<1 \mathrm{nM})$ and ligand $9($ IC50 < $1 \mathrm{nM})$, but also the influence of protein structure on those conformations. The analysis of the superimposed ligand structures when complexed with p38 $\alpha$ (Figure 5S) confirms that the common substructure has maintained its position, and shows the conformations adopted by Gleevec ${ }^{\circledR}$ and Nexavar $^{\circledR}$ in the experimental structures to be slightly different from the theoretical ones. These differences are higher for Nexavar ${ }^{\circledR}$, with the $\mathrm{N}$-methylformamide rotated $180^{\circ}$. Nevertheless, the $\mathrm{H}$-bond established between the nitrogen atom of the pyridine ring bound to $\mathrm{N}$-methylformamide and MET109 in the hinge region is established exactly the same way for both structures, while it does not seem that the $\mathrm{N}$ methylformamide establishes any interaction with the protein in any of the conformations.

The influence of the protein structure can be analysed by comparing the poses chosen for ligands 1,7 and 9 when docked into $p 38 \alpha$ Gleevec $^{\circledast}$, p38 $\alpha$ Nexavar ${ }^{\circledR}$ or $p 38 \alpha$ BIRB-796. As a general trend, the conformations adopted by the ligands only differ in that part of the ligands interacting with the hinge region. That region is formed by a loop with different structure for each of the three available protein structures. This can be explained as a consequence of the size of the ligand docked in each X-Ray structure. Thus, the conformation adopted by Gleevec ${ }^{\circledR}$ when docked into $p 38 \alpha$ Nexavar ${ }^{\circledR}$ scores the worst (Table 2 ), as a consequence of steric hindrance: in this case, the hinge region would clash with the pyridine ring because the hinge region was originally accommodating a smaller ligand. As for ligand 7 (see Figure 6S), we see that the hinge region is too far for $\mathrm{p} 38 \alpha$ Gleevec $^{\circledR}$ and the best interactions (with a H-bond with GLY110) are obtained for the p38 $\alpha$ BIRB-796 structure, which could be expected realising that the protein structure has the hinge region adapted for the morpholine ring shared by BIRB-796 and ligand 7. This trend is reproduced in Table 2, where ligand 7 is ranked best for p38 $\alpha$ BIRB-706.

All these results emphasize the importance of the hinge region of $p 38 \alpha$, even lacking a defined secondary structure. Thus, in order to take into account the structure of the hinge region, we will compare the predicted structure for ligand 1 docked into $p 38 \alpha$ Gleevec $^{\oplus}$, ligand 2 docked into p38 $\alpha$ Nexavar ${ }^{\circledast}$, ligand 3 docked into p38 $\alpha$ BIRB-796, ligand 7 docked into p38 $\alpha$ BIRB-796 structure, and ligand 9 docked into p38 $\alpha$ Nexavar ${ }^{\circledR}$ (Figure 7).

The experimental structures of Gleevec ${ }^{\circledast}$, Nexavar $^{\circledast}$ and BIRB-796 docked into $\mathrm{p} 38 \alpha[36,37]$ share an interaction with the hinge region through a $\mathrm{H}$-bond with MET109, and a H-bond network between ASP168...ligand...GLU71. As reported before for the B-Raf and C-Abl sets, our predicted structures suggest an extension of this H-bond network, this time just to the conserved LYS53 (see Figure 7S). Our modelled BIRB-796 ligand docked into p38 $\alpha$ allows us to propose a distinct feature, through a non-conserved TYR35 in the AP zone that expands that $\mathrm{H}$-bond network through a $\mathrm{H}$-bond with one of the urea NH groups. This implies that changing urea with acetamide would worsen its p38 $\alpha$ inhibition properties because another NH group is needed to establish a H-bond with GLU71. 


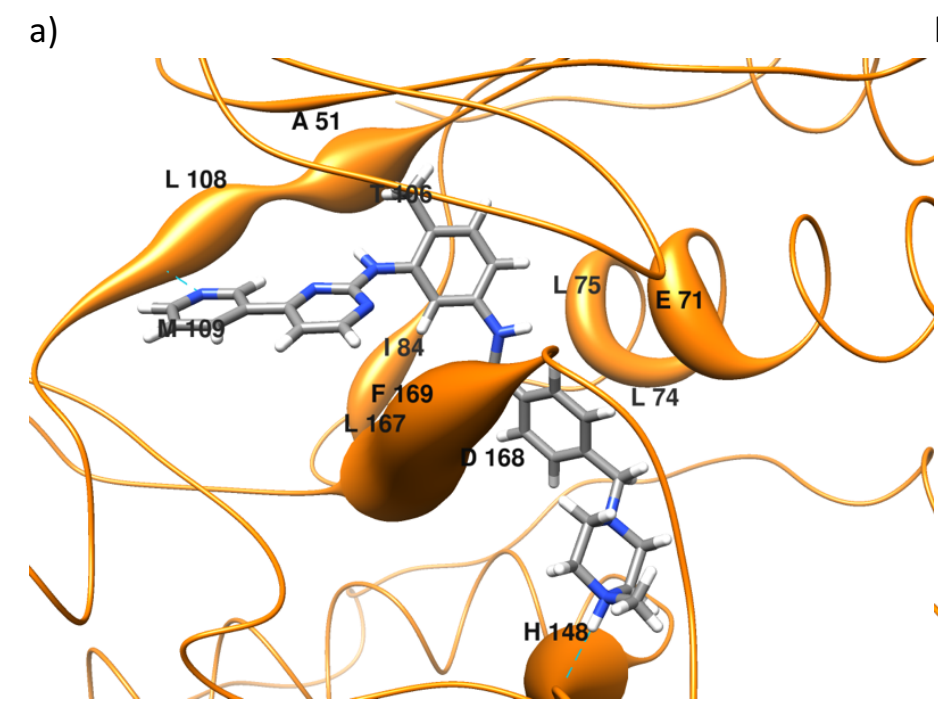

b)
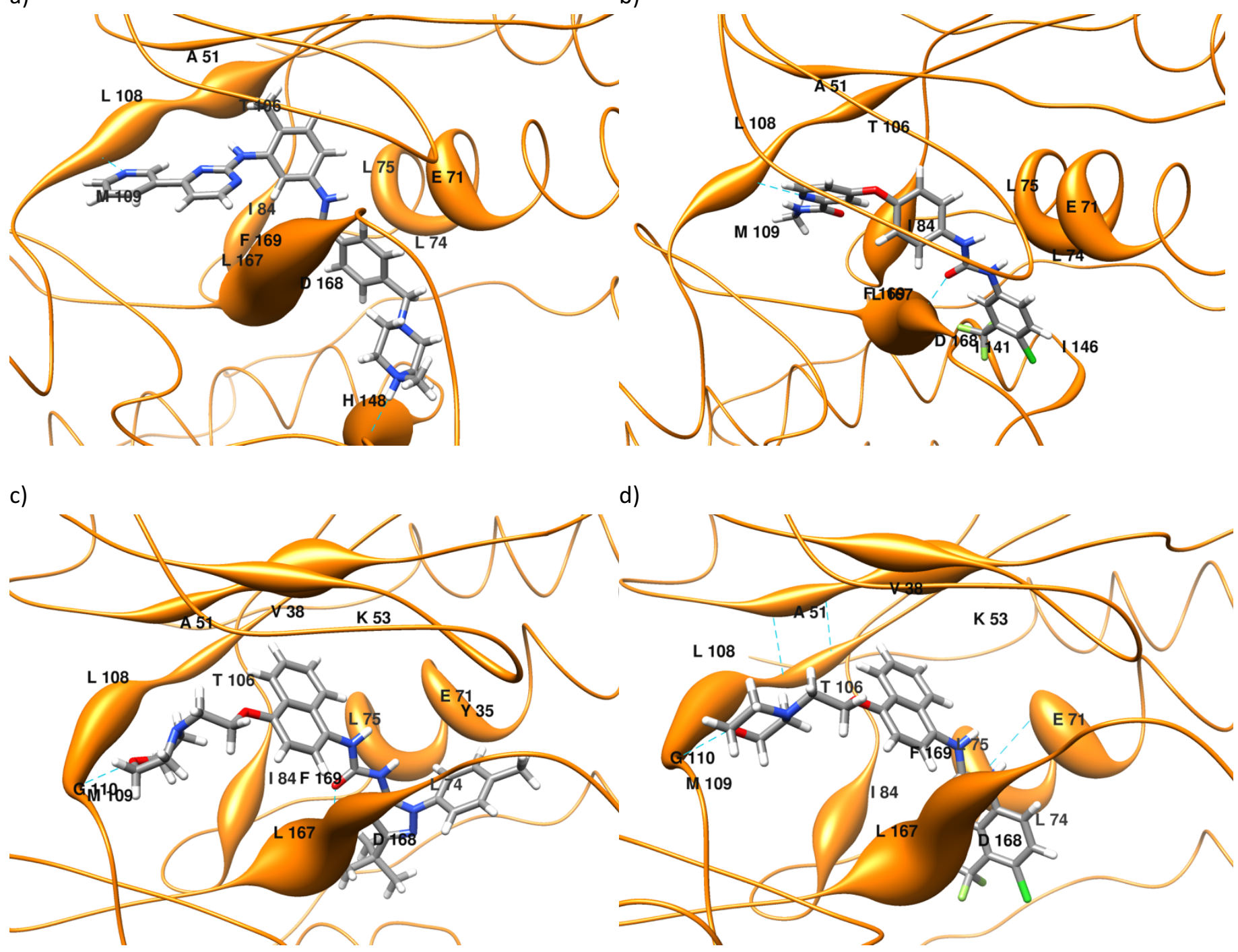

d)
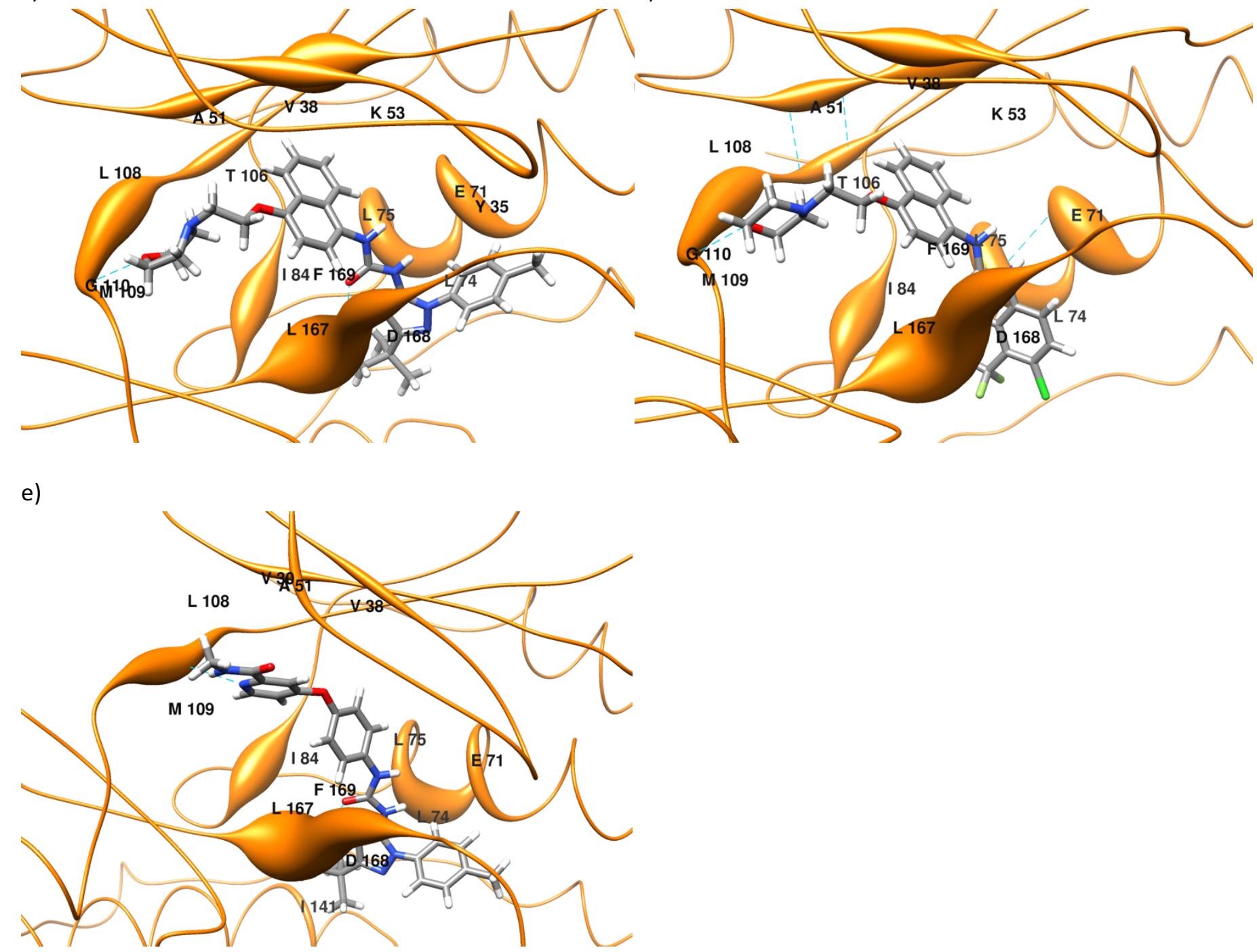

Figure 7: Theoretical structure for a) Gleevec ${ }^{\circledR}$, b) Nexavar ${ }^{\circledR}$, c) BIRB-796, d) ligand 7 and e) ligand 9, docked into $p 38 \alpha$ showing the protein with worm radii proportional to the MMGBSA predicted contribution of that residue to binding energy. 
An analysis from an energetic point of view (Figures 3 and 4) focus our attention on residues LYS53, THR106, HIS148 and LEU167. As for LYS53, differences arise from polar terms, so that the polar contribution of LYS53 for binding to BIRB-796 or ligand 7 favours binding, while for the other ligands it does not. This can be connected with the different conformation adopted by LYS53 and the DFG motif when ligands 3 and 7 are docked, compared to the conformation when the docked ligands are 1, 2 or 9. Figure 8, which compares those conformations for Gleevec ${ }^{\circledR}$ and ligand 7, shows how Gleevec ${ }^{\circledR}$ can establish more interactions with the DFG motif, although it seems that at cost of increasing the electrostatic repulsion with the ligand $\left(\Delta H_{\text {ele }}^{0}=12.9 \mathrm{kcal} \mathrm{mol}^{-1}\right.$ for Gleevec ${ }^{\circledast}$ versus $3.1 \mathrm{kcal} \mathrm{mol}^{-1}$ for BIRB-796).

THR106 establishes an already described H-bond with the ligand [36] (see Table 6S) that can explain its better polar contributions to binding. Differences for LEU167 seem mainly related to non-polar terms, while for HIS148 Gleevec ${ }^{\circledR}$ is the only ligand for which polar terms favour binding. This can be explained because the 1-methyl-piperazine ring in Gleevec ${ }^{\circledR}$ is able to establish favourable electrostatic interactions with HIS148 (-12.4 kcal mol ${ }^{-1}$ for Gleevec $^{\circledR}$ versus $1.34 \mathrm{kcal} \mathrm{mol}^{-1}$ for Nexavar$\left.{ }^{\circledR}\right)$. All these results point first to the need to investigate further on the dynamics of $p 38 \alpha$ to shed light into the importance of the LYS53 and hinge region conformations and how they adapt to the size of different ligands, which escapes the scope of the present work. Second, it seems that one of the reasons why the binding of Gleevec ${ }^{\circledR}$ to $\mathrm{p} 38 \alpha$ is weak is the use of acetamide instead of urea as linker. Finally, although the total positive charge of Gleevec ${ }^{\circledR}$ could be another of the reasons for its weak binding to $p 38 \alpha$, our results point to the need to explore the possibility of expanding the favourable interaction between the 1-methyl-piperazine ring and HIS148 with a non-charged heterocycle possessing a $\mathrm{H}$-bond donor feature.

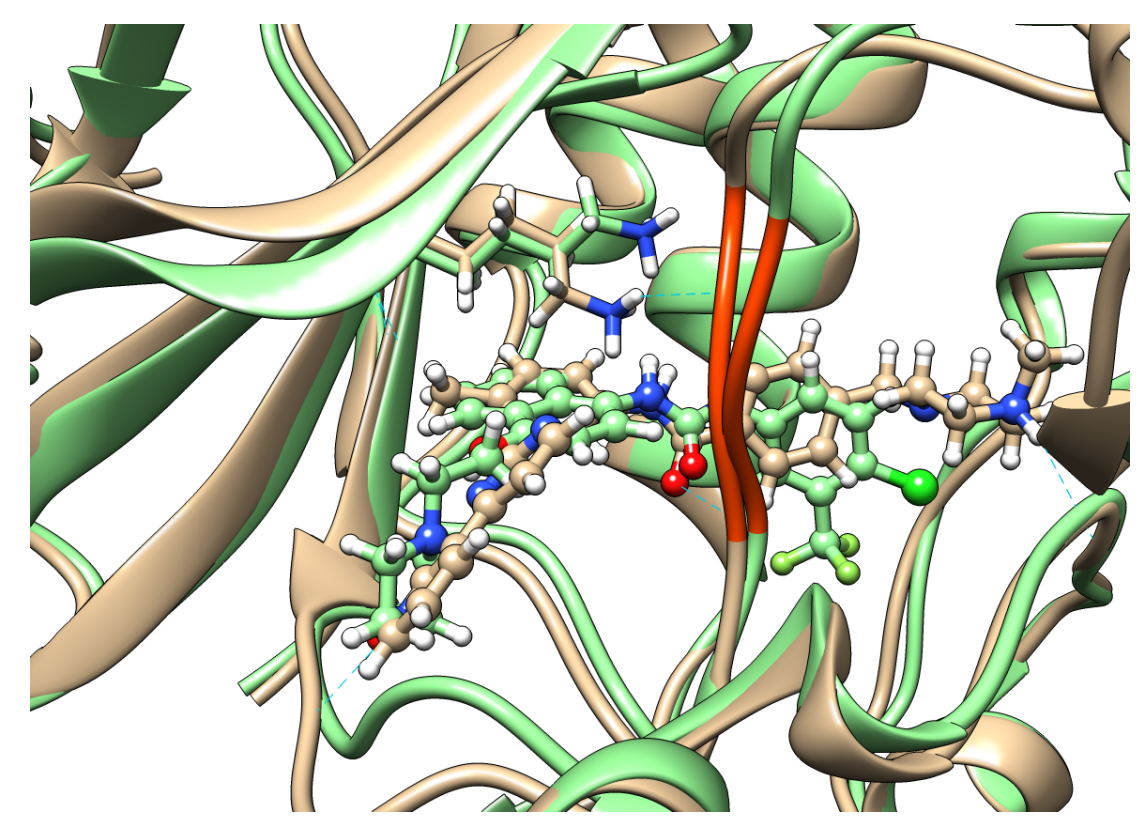

Figure 8: Superposition of complexes of $p 38 \alpha$ with Gleevec ${ }^{\circledR}$ (light brown) and ligand 7 (green) showing the different conformation adopted by LYS53 and the DFG motif (highlighted in dark orange)

\section{Conclusion}

This work analyses the possibility of improving a docking procedure specially designed for a congeneric series of compounds by means of a re-scoring of the initial binding poses through the MMPB(GB)SA methodology, essaying a set of different options within the methodology on an ensemble of eleven ligands bound to the B-Raf, c-Abl and p38 $\alpha$ kinases. Our results allow us to conclude that post processing is in general a good practice to be followed as it enhances the quality of the results at a relatively low computational cost. Although it is out of any doubt that a post processing using MMPB(GB)SA with a long MD trajectory is expected to give better results in a broad range of situations, it is clear also from our results that a rational comparison of quicker options, having in mind that results are case-dependent, can improve these results requiring much less resources. This can be of great importance when the set to be analysed is big. In this sense, for the sets studied in this work, short MD runs do not always provide better results, which also 
happens with the inclusion of entropy. As the treatment of solvent is concerned, there is consensus in that $G B=2$ is better than $\mathrm{GB}=5$, and also in that a previous analysis of different options for $\varepsilon_{\mathrm{i}}$ is advisable.

The analysis of the different scoring parameters obtained has allowed us to choose the best method for each of the studied proteins. The total charge of the proteins studied (B-Raf $+9, c-A b l-6$ and $p 38 \alpha-3$ ) and the binding strength of positively charged ligands can be correlated to propose as a qualitative rule that positively charged weak binders need a high dielectric constant to be correctly scored when binding to a positively charged protein, while they need a low dielectric constant when binding to a negatively charged protein. We would thus propose as an a priori protocol to use the one-snapshot MMPBSA post-processing methodology, with appropriate $\varepsilon_{\mathrm{i}}$ and no entropic term. Hence, we have analysed the proposed docked structures from structural and energetic points of view focussing in those ligands that are predicted experimentally to bind strongly to each of the proteins, in order to discern which features are required to propose further modifications that would improve them. Thus, for B-Raf we suggest that ligand 7 with an heteroaromatic moiety instead of the morpholine ring would be even more active, while a modification of Gleevec ${ }^{\circledR}$ changing the acetamide linker with a urea type one would improve its c-Abl inhibition. Finally, our protocol allows us to conclude that the urea linker is also important for p38 $\alpha$ inhibition, and that the possibility of changing the 1-methylpiperazine ring with a non-charged heterocycle that can donor a H-bond to HIS148 could be an interesting route to be explored.

\section{Future perspective}

There exists a clear interest in develop new kinase inhibitors. The increase in available simulation timescales through potent GPUs together with new sampling methodologies like Gaussian Accelerated Molecular Dynamics are expected to find new allosteric pockets that will allow researchers to propose potent type III kinase inhibitors.

\section{Summary points}

- Re-scoring after a docking procedure is a computationally cheap and useful methodology to improve the quality of results

- Using just one snapshot within the MMPB(GB)SA methodology, together with a rational comparison of different parameters, could be as useful as a long MD run

- When the set to be studied is big an a priori protocol would be to use one snapshot MMPBSA with no entropic term and choose $\varepsilon_{\mathrm{i}}$ according to the charge of protein and ligands

- B-Raf could be increased modifying ligand 7 with an heteroaromatic moiety instead of the morpholine ring

- c-Abl inhibition could be increased modifying Gleevec ${ }^{\circledR}$ by changing the acetamide linker with a urea type one

- $\quad$ p38 $\alpha$ inhibition could be increased by changing the 1-methyl-piperazine ring with a non-charged heterocycle being able to establish an $\mathrm{H}$-bond with HIS148

\section{References}

1. Manning G, Whyte DB, Martinez R, Hunter T, Sudarsanam S. The Protein Kinase Complement of the Human Genome. Science 298(5600), 1912-1934 (2002).

2. Marino KA, Sutto L, Gervasio FL. The Effect of a Widespread Cancer-Causing Mutation on the Inactive to Active Dynamics of the B-Raf Kinase. JACS 137(16), 5280-5283 (2015).

3. Zhang J, Yang PL, Gray NS. Targeting cancer with small molecule kinase inhibitors. Nat. Rev. Cancer 9(1), 2839 (2009).

** Describes the role of kinases in cancer and the different types of inhibitors

4. Garuti L, Roberti M, Bottegoni G. Non-ATP Competitive Protein Kinase Inhibitors. Curr. Med. Chem. 17(25), 2804-2821 (2010).

5. Backes AC, Zech B, Felber B, Klebl B, Müller G. Small-molecule inhibitors binding to protein kinase. Part II: the novel pharmacophore approach of type II and type III inhibition. Expert Opinion on Drug Discovery 3(12), 1427-1449 (2008).

6. Fasano M, Della Corte CM, Califano R et al. Type III or allosteric kinase inhibitors for the treatment of nonsmall cell lung cancer. Expert Opinion on Investigational Drugs 23(6), 809-821 (2014).

7. Tsai CJ, Nussinov R. A unified view of "how allostery works". PLoS Comp. Biol. 10(2), e1003394 (2014).

** Very useful to understand allostery 
8. Liu Y, Gray NS. Rational design of inhibitors that bind to inactive kinase conformations. Nat. Chem. Biol. 2(7), 358-364 (2006).

9. Matthew RL, Celia D. MAP Kinase p38Inhibitors: Clinical Results and an Intimate Look at Their Interactions with p38\&\#945; Protein. Curr. Med. Chem. 12(25), 2979-2994 (2005).

10. Dietrich J, Hulme C, Hurley LH. The design, synthesis, and evaluation of 8 hybrid DFG-out allosteric kinase inhibitors: A structural analysis of the binding interactions of Gleevec ${ }^{\circledR}$, Nexavar ${ }^{\circledR}$, and BIRB-796. Biorg. Med. Chem. 18(15), 5738-5748 (2010).

* The article which has inspired us to prepare this one

11. Warren GL, Andrews CW, Capelli A-M et al. A Critical Assessment of Docking Programs and Scoring Functions. J. Med. Chem. 49(20), 5912-5931 (2006).

12. Cheng T, Li X, Li Y, Liu Z, Wang R. Comparative Assessment of Scoring Functions on a Diverse Test Set. J. Chem. Inf. Mod. 49(4), 1079-1093 (2009).

13. Rapp C, Kalyanaraman C, Schiffmiller A, Schoenbrun EL, Jacobson MP. A Molecular Mechanics Approach to Modeling Protein-Ligand Interactions: Relative Binding Affinities in Congeneric Series. J. Chem. Inf. Mod. 51(9), 2082-2089 (2011).

14. Thompson DC, Humblet C, Joseph-Mccarthy D. Investigation of MM-PBSA Rescoring of Docking Poses. J. Chem. Inf. Mod. 48(5), 1081-1091 (2008).

15. Hou T, Wang J, Li Y, Wang W. Assessing the performance of the MM/PBSA and MM/GBSA methods: II. The accuracy of ranking poses generated from docking. J. Comput. Chem. 32(5), 866-877 (2011).

16. Kollman PA, Massova I, Reyes C et al. Calculating Structures and Free Energies of Complex Molecules: Combining Molecular Mechanics and Continuum Models. Acc. Chem. Res. 33(12), 889-897 (2000).

* Describes the MMPB(GB)SA methodology

17. Rastelli G, Rio AD, Degliesposti G, Sgobba M. Fast and accurate predictions of binding free energies using MM-PBSA and MM-GBSA. J. Comput. Chem. 31(4), 797-810 (2010).

18. Kuhn B, Gerber P, Schulz-Gasch T, Stahl M. Validation and Use of the MM-PBSA Approach for Drug Discovery. J. Med. Chem. 48(12), 4040-4048 (2005).

19. Genheden S, Ryde U. Comparison of end-point continuum-solvation methods for the calculation of proteinligand binding free energies. Proteins: Struct. Funct. Bioinform. 80(5), 1326-1342 (2012).

20. Warshel A, Sharma PK, Kato M, Parson WW. Modeling electrostatic effects in proteins. Biochim. Biophys. Acta 1764(11), 1647-1676 (2006).

21. Schutz CN, Warshel A. What are the dielectric "constants" of proteins and how to validate electrostatic models? Proteins: Struct. Funct. Bioinform. 44(4), 400-417 (2001).

22. Li L, Li C, Zhang Z, Alexov E. On the Dielectric "Constant" of Proteins: Smooth Dielectric Function for Macromolecular Modeling and Its Implementation in DelPhi. Journal of Chemical Theory and Computation 9(4), 2126-2136 (2013).

23. Genheden S, Ryde U. The MM/PBSA and MM/GBSA methods to estimate ligand-binding affinities. Expert Opinion on Drug Discovery 10(5), 449-461 (2015).

24. Yang T, Wu JC, Yan C et al. Virtual screening using molecular simulations. Proteins: Struct. Funct. Bioinform. 79(6), 1940-1951 (2011).

25. Luis C, Jose MG-R, Marta P, Maria ST, Maria DP, Jaime R-M. Insight into the Binding of DFG-out Allosteric Inhibitors to B-Raf Kinase Using Molecular Dynamics and Free Energy Calculations. Curr. Comput. Aided Drug Des. 11(2), 124-136 (2015).

26. Hornak V, Abel R, Okur A, Strockbine B, Roitberg A, Simmerling C. Comparison of multiple Amber force fields and development of improved protein backbone parameters. Proteins: Struct. Funct. Bioinform. 65(3), 712725 (2006).

27. Wang J, Wolf RM, Caldwell JW, Kollman PA, Case DA. Development and testing of a general amber force field. J. Comput. Chem. 25(9), 1157-1174 (2004).

28. Case DA, Babin V, Berryman JT et al. AMBER 14. University of California, San Francisco, University of California, San Francisco. doi:citeulike-article-id:5692441(2014).

29. Darden T, York D, Pedersen L. Particle mesh Ewald: An N. $\log (\mathrm{N})$ method for Ewald sums in large systems. The Journal of chemical physics 98(12), 10089-10092 (1993).

30. Jorgensen WL, Chandrasekhar J, Madura JD, Impey RW, Klein ML. Comparison of simple potential functions for simulating liquid water. J. Chem. Phys. 79(2), 926-935 (1983).

31. Pettersen EF, Goddard TD, Huang CC et al. UCSF Chimera-A visualization system for exploratory research and analysis. J. Comput. Chem. 25(13), 1605-1612 (2004).

32. Origin. OriginLab, Northampton, MA. 
33. Berman HM, Westbrook J, Feng Z et al. The Protein Data Bank. Nucleic Acids Res. 28(1), 235-242 (2000).

34. Nagar B, Bornmann WG, Pellicena P et al. Crystal Structures of the Kinase Domain of c-Abl in Complex with the Small Molecule Inhibitors PD173955 and Imatinib (STI-571). Cancer Res. 62(15), 4236-4243 (2002).

35. Wan PTC, Garnett MJ, Roe SM et al. Mechanism of Activation of the RAF-ERK Signaling Pathway by Oncogenic Mutations of B-RAF. Cell 116(6), 855-867 (2004).

36. Namboodiri HV, Bukhtiyarova M, Ramcharan J, Karpusas M, Lee Y, Springman EB. Analysis of Imatinib and Sorafenib Binding to $\mathrm{p} 38 \alpha$ Compared with c-Abl and b-Raf Provides Structural Insights for Understanding the Selectivity of Inhibitors Targeting the DFG-Out Form of Protein Kinases. Biochemistry 49(17), 3611-3618 (2010).

37. Pargellis $C$, Tong L, Churchill L et al. Inhibition of p38 MAP kinase by utilizing a novel allosteric binding site. Nat. Struct. Mol. Biol. 9(4), 268-272 (2002).

38. Gill AL, Frederickson M, Cleasby A et al. Identification of Novel p38 $\alpha$ MAP Kinase Inhibitors Using FragmentBased Lead Generation. J. Med. Chem. 48(2), 414-426 (2005).

39. Jakalian A, Bush BL, Jack DB, Bayly Cl. Fast, efficient generation of high-quality atomic charges. AM1-BCC model: I. Method. J. Comput. Chem. 21(2), 132-146 (2000).

40. Jakalian A, Jack DB, Bayly Cl. Fast, efficient generation of high-quality atomic charges. AM1-BCC model: II. Parameterization and validation. J. Comput. Chem. 23(16), 1623-1641 (2002).

41. Molecular Operating Environment (MOE) 2013.08. Chemical Computing Group Inc., 1010 Sherboke St. West, Suite \#90, Montreal, QC, Canada, H3A 2R7, 2013. (2013.08).

42. Miller BR, Mcgee TD, Swails JM, Homeyer N, Gohlke H, Roitberg AE. MMPBSA.py: An Efficient Program for End-State Free Energy Calculations. Journal of Chemical Theory and Computation 8(9), 3314-3321 (2012).

43. Rocchia W, Alexov E, Honig B. Extending the Applicability of the Nonlinear Poisson-Boltzmann Equation: Multiple Dielectric Constants and Multivalent Ions. The Journal of Physical Chemistry B 105(28), 6507-6514 (2001).

44. Onufriev A, Bashford D, Case DA. Exploring protein native states and large-scale conformational changes with a modified generalized born model. Proteins: Struct. Funct. Bioinform. 55(2), 383-394 (2004).

45. Fieller EC, Hartley HO, Pearson ES. TESTS FOR RANK CORRELATION COEFFICIENTS. I. Biometrika 44(3-4), 470481 (1957).

46. Foloppe N, Hubbard R. Towards Predictive Ligand Design With Free-Energy Based Computational Methods? Curr. Med. Chem. 13(29), 3583-3608 (2006).

47. Wang W, Lim WA, Jakalian A et al. An Analysis of the Interactions between the Sem-5 SH3 Domain and Its Ligands Using Molecular Dynamics, Free Energy Calculations, and Sequence Analysis. JACS 123(17), 39863994 (2001).

48. Singh N, Warshel A. Absolute binding free energy calculations: On the accuracy of computational scoring of protein-ligand interactions. Proteins: Struct. Funct. Bioinform. 78(7), 1705-1723 (2010).

49. Junmei W, Tingjun H, Xiaojie X. Recent Advances in Free Energy Calculations with a Combination of Molecular Mechanics and Continuum Models. Curr. Comput. Aided Drug Des. 2(3), 287-306 (2006).

50. Yang Y, Shen Y, Liu H, Yao X. Molecular Dynamics Simulation and Free Energy Calculation Studies of the Binding Mechanism of Allosteric Inhibitors with p38 a MAP Kinase. J. Chem. Inf. Mod. 51(12), 3235-3246 (2011).

51. Schindler T, Bornmann W, Pellicena P, Miller WT, Clarkson B, Kuriyan J. Structural Mechanism for STI-571 Inhibition of Abelson Tyrosine Kinase. Science 289(5486), 1938-1942 (2000).

52. Liu F, Wang B, Wang Q et al. Discovery and characterization of a novel potent type II native and mutant BCRABL inhibitor (CHMFL-074) for Chronic Myeloid Leukemia (CML). Oncotarget; Vol 7, No 29 (2016). 